\title{
Rotation and differential rotation in field F- and G-type stars ${ }^{\star \star \star}$
}

\author{
A. Reiners ${ }^{1}$ and J. H. M. M. Schmitt ${ }^{1}$ \\ Hamburger Sternwarte, Universität Hamburg, Gojenbergsweg 112, 21029 Hamburg, Germany \\ Received 2 October 2002 / Accepted 3 November 2002

\begin{abstract}
We present a detailed study of rotation and differential rotation analyzing high resolution high $S / N$ spectra of 142 F-, G- and early K-type field stars. Using Least Squares Deconvolution we obtain broadening profiles for our sample stars and use the Fourier transform method to determine projected rotational velocities $v \sin i$. Distributions of rotational velocities and periods are studied in the HR-diagram. For a subsample of 32 stars of spectral type F0-G0 we derive the amount of differential rotation in terms of $\alpha=\left(\Omega_{\text {Equator }}-\Omega_{\text {Pole }}\right) / \Omega_{\text {Equator }}$. We find evidence for differential rotation in ten of the 32 stars. Differential rotation seems to be more common in slower rotators, but deviations from rigid rotation are also found in some fast rotators. We search for correlations between differential rotation and parameters relevant for stellar activity and show indications against strong differential rotation in very active stars. We derive values of $\Delta P$ and $\Delta \Omega$, which support a period dependence of differential rotation. Derived lap times $2 \pi / \Delta \Omega$ are of the order of $20 \mathrm{~d}$ and contradict the assumption that constant lap times of the order of the solar one $(\sim 130 \mathrm{~d})$ are the rule in stars that are thought to harbour magnetic dynamos.
\end{abstract}

Key words. stars: rotation - stars: late-type - stars: activity

\section{Introduction}

Stellar surface phenomena are driven by a variety of different forces. Gradients of temperature and gravity together with different ionization stages determine the depth of surface convection zones. Stellar rotation implies Coriolis forces that interact with turbulent fluid motions. Especially interesting are the processes taking place in the presence of magnetic fields; differential rotation is expected to wind up magnetic field lines and to maintain a stellar dynamo. Such dynamos are believed to be the cause of the plethora of activity phenomena like, e.g., spots, chromosperic CarI emission, X-ray emission and activity cycles. As a consequence stars are generally not expected to rotate rigidly and different kinds of rotation laws can be imagined even in the absence of magnetic fields.

The only direct evidence for differential rotation comes from spatially resolved observations of the Sun; the solar rotation law can be approximated as

$\Omega(l)=\Omega_{\text {Equator }}\left(1-\alpha \sin ^{2} l\right)$,

with $l$ being the latitude and $\alpha_{\odot} \approx 0.2$, i.e., the Equator rotating $20 \%$ faster than the Pole.

Send offprint requests to: A. Reiners,

e-mail: areiners@hs.uni-hamburg.de

* Based on observations collected at the European Southern Observatory, La Silla.

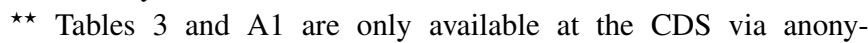
mous ftp to cdsarc.u-strasbg.fr $(130.79 .125 .5)$ or via http://cdsweb.u-strasbg.fr/cgi-bin/qcat?J/A+A/398/647
Stellar differential rotation cannot be directly measured. Three main methods have been used for the search for deviations from rigid rotation on stars other than the Sun. (i) Variations of photometric periods, e.g., in chromospheric CaII emission (Donahue et al. 1996) or broadband photometry (Hall 1991). (ii) Identifying individual features on Doppler maps and follow their migration with time (e.g., Donati \& Collier Cameron 1997; Barnes et al. 2000). (iii) Studying line profiles (Gray 1977, 1982; Wöhl 1983; Reiners et al. 2001). In this paper we will pursue method (iii).

Searching for non-rigid rotation using method (iii), it is crucial to disentangle the different stellar velocity fields. Realizing this, Gray (1973) examined the Fourier transform of stellar absorption profiles. Utilizing the fact that convolutions become multiplications in Fourier domain, he showed that velocity fields like micro- and macroturbulence and especially rotation are indeed distinguishable in the Fourier domain. However, no examples of non-rigid rotation were found among A-type (Gray 1977) and F-type (Gray 1982) stars.

For a determination of the rotation law the zeros of the Fourier transformed broadening profile are of particular interest. The ratio of the first and second zeros is a direct indicator for solar-like differential rotation (Reiners \& Schmitt 2002a), i.e., $\alpha>0$; equatorial regions rotate faster than polar ones. The important point is that the zeros of a Fourier transform are unaffected by multiplications with other velocity profiles. As long as other line broadening effects do not introduce additionally zeros - and convolutions are appropriate approximations of the superposition of the different velocity fields - the rotation law 

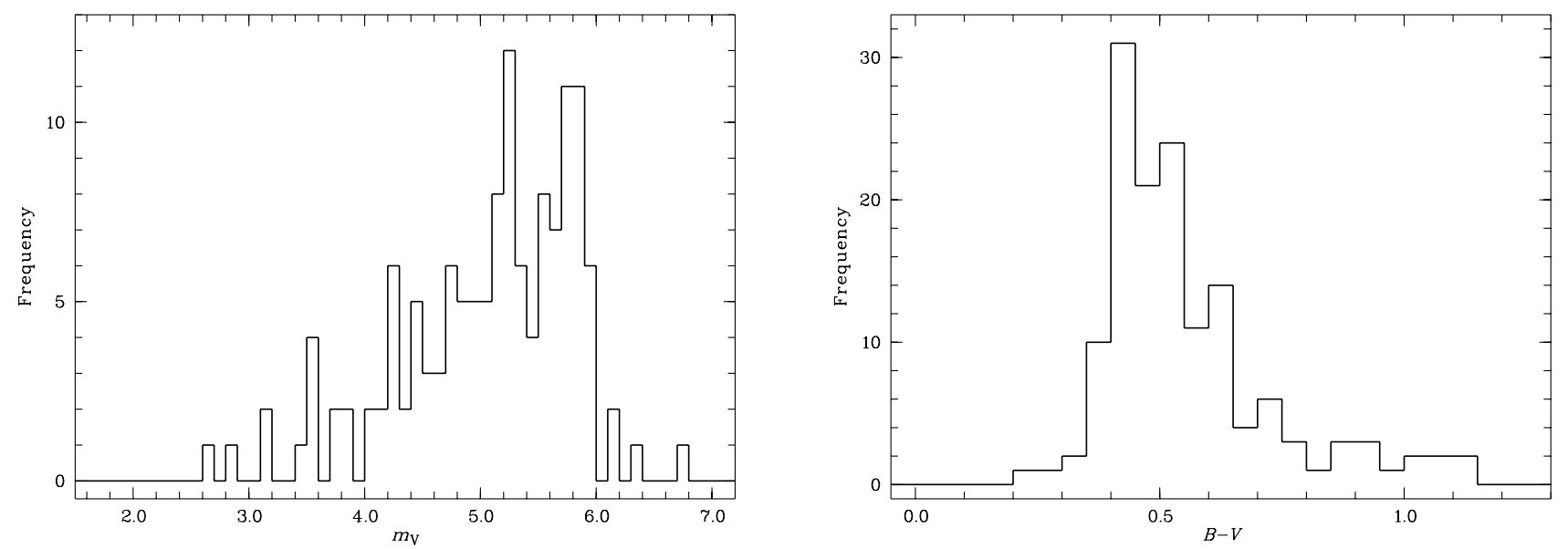

Fig. 1. Distributions of our primary data sample of 142 F-, G- and K-stars in apparent magnitude ( $m_{V}$, left) and $B-V$ colour (right).

Table 1. Observations.

\begin{tabular}{rccc}
\hline \hline Date & \# Objects & Region & Resolution \\
\hline 13.10 .2000 & 11 & $5770-5810 \AA$ & 235000 \\
$01 .-04.10 .2001$ & 62 & $5770-5810 \AA$ & 235000 \\
$01 .-03.04 .2002$ & 77 & $6225-6270 \AA$ & 235000 \\
\hline
\end{tabular}

can be directly measured in the Fourier transform of stellar absorption profiles without any need of modelling.

In the present paper we apply the Fourier transform method (FTM) to a sample of late type stars with moderate rotational velocities $\left(v \sin i \leq 45 \mathrm{~km} \mathrm{~s}^{-1}\right)$. We derive projected rotational velocities $v \sin i$ for all of our sample stars. The measurement of differential rotation in terms of $\alpha$ is possible only for a subsample (Sect. 3); for an overview on the method and the required data quality we refer to Reiners \& Schmitt (2002a,b). We present a relation between the observable ratio of the zero positions in the Fourier domain and the differential rotation parameter $\alpha$ on the basis of model calculations. Finally, we search for correlations between our derived values of $\alpha$ and other stellar parameters, focusing on those believed to be relevant for stellar activity.

\section{Observations}

Our primary data sample consists of 142 F-, G- and K-dwarfs visible from ESO La Silla, Chile. Histograms in visual magnitude and $B-V$ colour characterizing our sample are shown in Fig. 1. We concentrated on stars with known rotational velocity in the range $10 \mathrm{~km} \mathrm{~s}^{-1}<v \sin i<40 \mathrm{~km} \mathrm{~s}^{-1}$. However, we also observed 30 stars without known rotational velocities.

The data was taken with CES at the ESO $3.6 \mathrm{~m}$ telescope at La Silla, Chile. In its highest resolution mode $(R=235000)$ the spectral region covers $\sim 40 \AA$. In the three observing runs listed in Table 1 we used two different wavelength regions at 5770-5810 $\AA$ and 6225-6270 A. For a detection of the subtle effects of differential rotation high $S / N$-ratios are essential. The $S / N$-ratios of our data range between 400 and 900 for all observations. As an example of the quality of our data we show two spectra in the region $6225-6270 \AA$ in Fig. 2. To study the effects of different wavelength regions, seven stars were observed both in the 5770-5810 $\AA$ and in the $6225-6270 \AA$ regions. $\psi$ Cap was observed in both runs where the 5770-5810 $\stackrel{\circ}{\text { region was }}$ used.

\section{Extraction of the broadening profiles and the Fourier Transform Method}

With the Fourier Transform Method (FTM), differential rotation can be measured in stars with $v \sin i \gtrsim 10 \mathrm{~km} \mathrm{~s}^{-1}$ (for an introduction to the method see Reiners \& Schmitt 2002a,b). For stars with lower rotational velocity other line broadening effects become dominant and the measurement of differential rotation is not reliable. For the slowest rotators with narrow spectral lines high resolution is required. To detect differential rotation on stars with $v \sin i<20 \mathrm{~km} \mathrm{~s}^{-1}$, spectra with a resolution of $R \geq 100000$ are needed.

To measure the subtle effects of differential rotation on the blended spectral lines it is necessary to achieve extremely high $S / N$-ratios. Therefore we extract an overall broadening profile for each star using as many absorption lines as possible by carrying out a "Physical Least Squares Deconvolution" (PLSD) process: we constructed an unbroadened $\delta$-template using a slowly rotating star of similar spectral type as a reference. Line positions are compared with data from the Vienna Atomic Line Database (Kupka et al. 1999) and only well determined lines are used. Central wavelengths were optimized on the reference spectrum to correct for convective wavelength shifts. With such a template as a starting point we fitted the convolution of the template and a broadening profile to the data by alternately optimizing the equivalent widths of the individual template lines and the shape of the broadening profile. The broadening profile was parameterized using Chebychev polynomials. 25 polynomials were used to allow for small-scale variations. For line profiles of stars rotating as slowly as our sample stars thermal broadening becomes important. Thus after the iteration we broadened the template lines according to the effective temperature of the star and the atomic weight of the absorbing atom. With that template we finally deconvolved the overall broadening profile. 


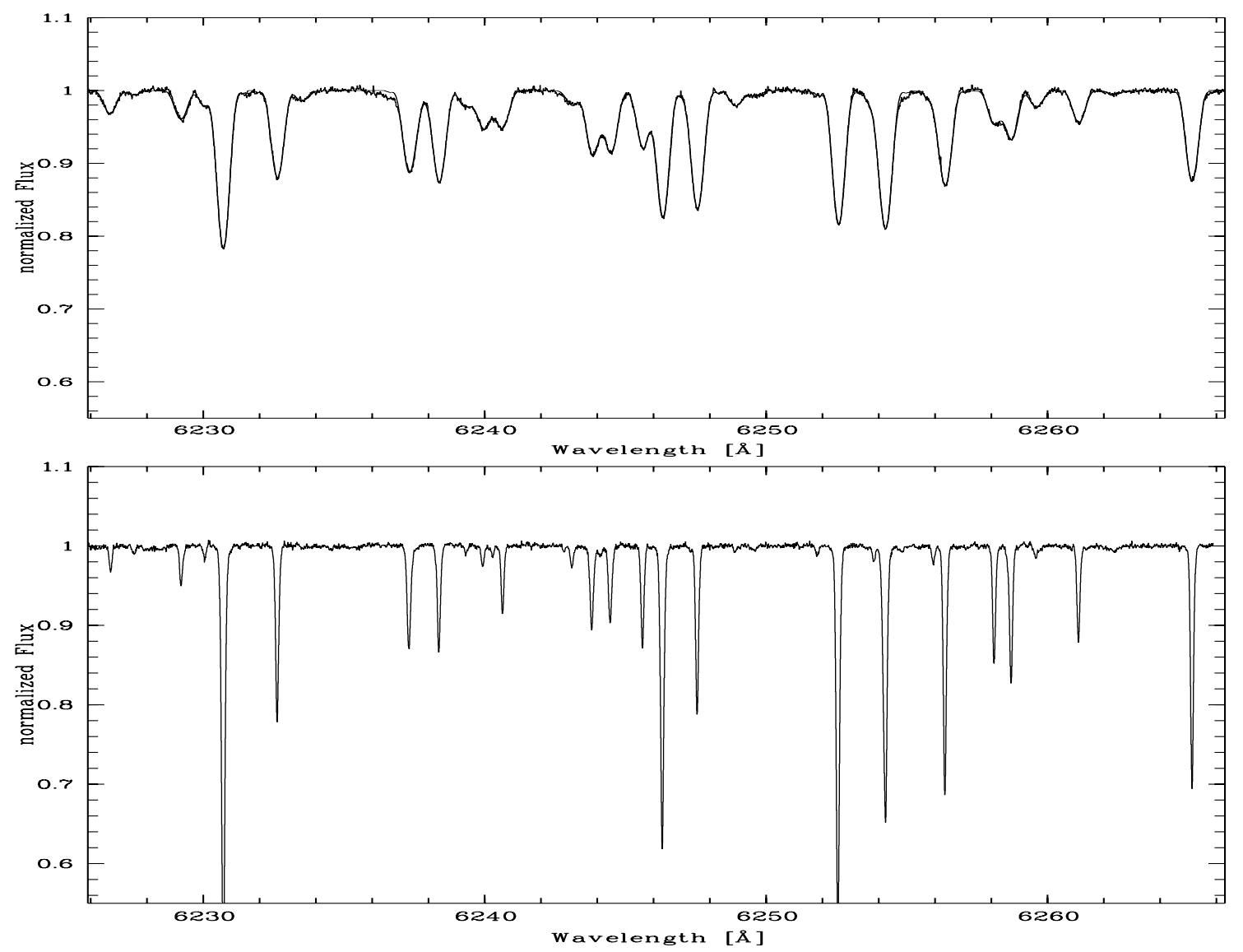

Fig. 2. Two spectra in the region 6225-6270 Å. Top panel: the "fast" rotator HD $89449\left(v \sin i=17.4 \mathrm{~km} \mathrm{~s}^{-1}\right)$. The convolution of our derived broadening profile and the optimized line template is overplotted (cf. Sect. 3); bottom panel: slow rotator HD $76932\left(v \sin i=2.6 \mathrm{~km} \mathrm{~s}^{-1}\right)$. For the slow rotators no broadening profile was derived and only the data is plotted here.

In order to provide an impression of the quality of our broadening profiles, we convolved the final template with the final broadening profile. Some results can be seen in the top panel of Fig. 2 and in Figs. 10b, d, f. Solid lines indicate the results of the convolutions that are plotted over the error bars of the data. Especially in Fig. 2 the quality of the fit over the whole spectral region is apparent.

Our sample also contains a number of spectroscopic binaries and stars with peculiar profiles for which no broadening profiles could be derived. Those spectra have been discarded and no measurement of differential rotation was possible for them. For the slowest rotators $\left(v \sin i \lesssim 6 \mathrm{~km} \mathrm{~s}^{-1}\right)$ no useful derivation of a broadening profile was possible either. Since no differential rotation can be derived for those stars anyway, we calculated the projected rotational velocity $v \sin i$ from a mean profile of the absorption lines due to the heaviest ions with the smallest intrinsic widths. Each absorption line used is transformed into velocity space and the results are co-added. The value of $v \sin i$ was then derived from that mean profile (see Sect. 4).

If the derivation of an overall broadening profile is successful, peculiarities like asymmetries or spectroscopic duplicity of the stars can easily be found given the obtained quality of the profile. For the detection of differential rotation with FTM profile symmetry is essential, since large-scale turbulence or photospheric distortions like spots can seriously disturb the signal of the underlying rotational broadening profile. Before Fourier transform apparently symmetric profiles of the fast rotators have been tested for asymmetry by mirroring the profiles at the center and taking the average of both prototypes. We qualified a profile as symmetric if the averaged profile falls completely within the error bars of the original line. Examples of symmetric and asymmetric profiles are shown in Figs. 3 and 4.

The symmetric broadening profiles are Fourier transformed and the first two zeros, $q_{1}$ and $q_{2}$, of the Fourier transform are measured. Both the projected rotational velocity $v \sin i$ and the differential rotation parameter $\alpha$ - assuming a solar-like rotation law - are determined by the zeros of the Fourier profiles. The absolute positions of the zeros are determined by the value of $v \sin i$ (see Sect. 4), and the rotational broadening law defines the ratio $q_{2} / q_{1}$ as shown in Reiners \& Schmitt (2002a) (see Sect. 6).

For slowly rotating stars the signal vanishes in the noise and $q_{2}$ cannot be measured. In addition to the principal limit of detecting differential rotation with FTM to stars with projected rotational velocities of $v \sin i \gtrsim 10 \mathrm{~km} \mathrm{~s}^{-1}$ an additional threshold due to the limited $S / N$-ratio is inferred. Calculations of that limit can be found in Reiners \& Schmitt (2002c), in our case the limit applies at about $v \sin i \gtrsim 12 \mathrm{~km} \mathrm{~s}^{-1}$. 

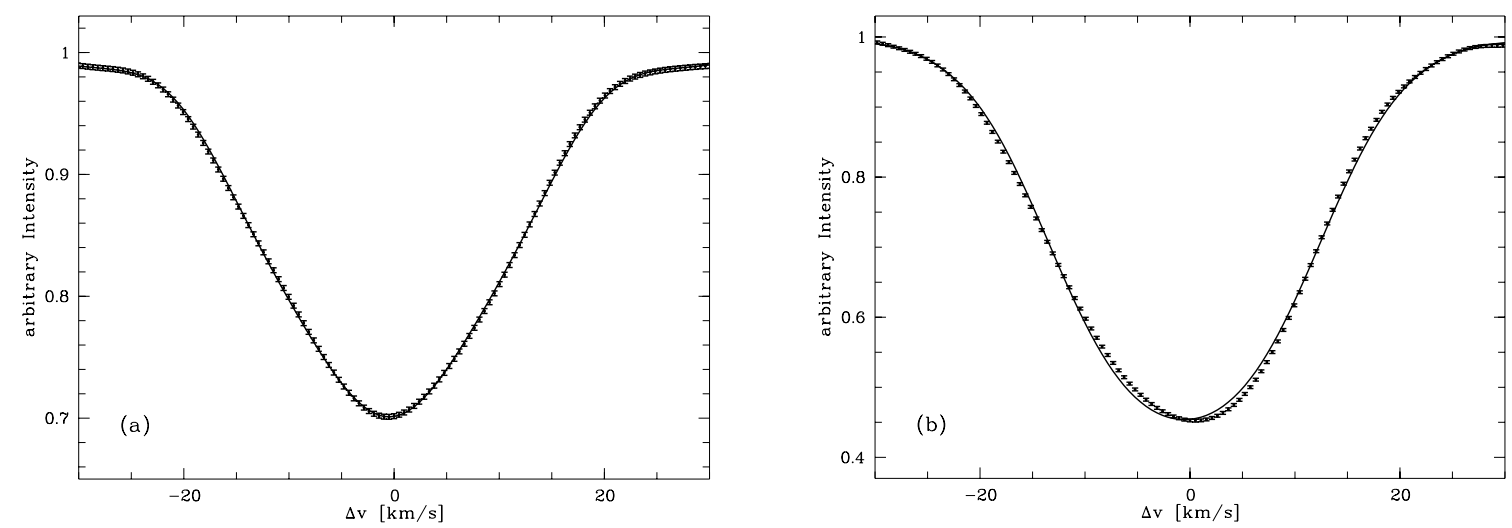

Fig. 3. Example of a) a symmetric (HD 173 667) and b) an asymmetric (HD 98 991) broadening profile. The symmetrized broadening profiles are plotted over the error bars of the data (see text).
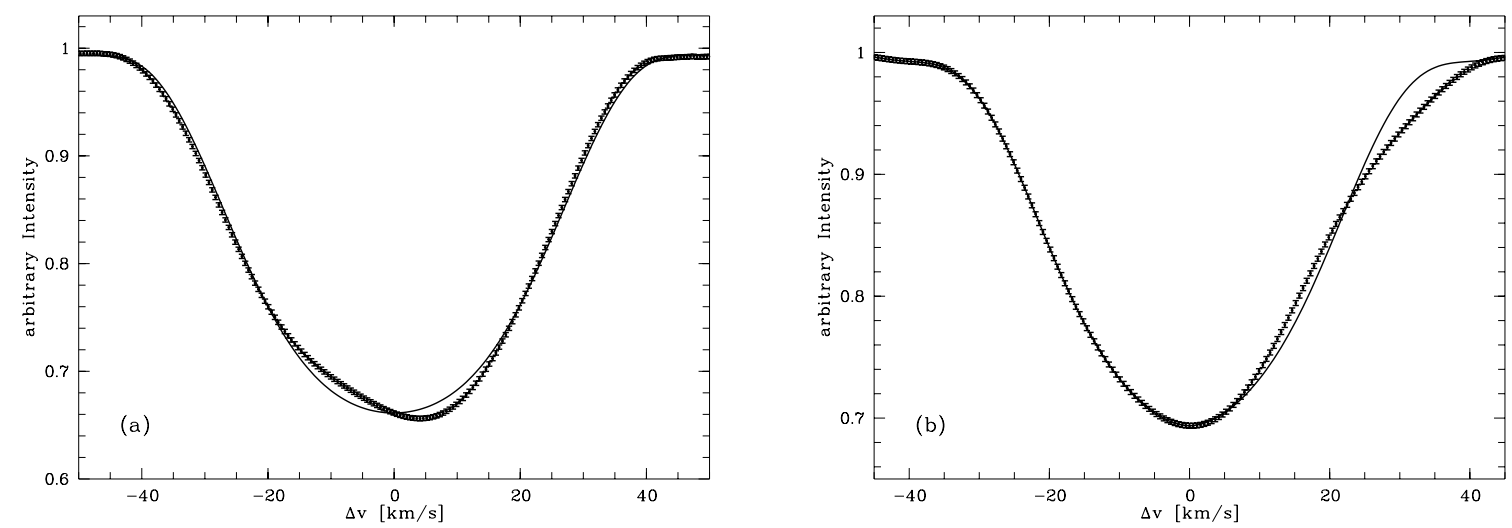

Fig. 4. Asymmetric mean profiles of a) HD 118646 and b) HD 110379 . In a) the line core is strongly asymmetric (the symmetrized broadening profiles is plotted over the data). In b) the core is symmetric and the blue and red flank differ, here we mirrored the left flank at the line center and plotted the mirrored profile over the data.

No asymmetries are expected in the profiles from rotationally line broadening and for our determination of the rotation law only the real Fourier components can be used. Thus we averaged out small asymmetries in the profiles as explained above and used the averaged profile to calculate the Fourier transform. Simulations showed that the asymmetries we allow in our procedure lead to deviations in $q_{2} / q_{1}$ that are well contained within the calculated errors due to limited $S / N$-ratio.

In case of asymmetry we transformed the original unsymmetrized broadening profile and determined only the projected rotational velocity $v \sin i$, a procedure that is relatively stable against asymmetries within the lines. No value of $q_{2} / q_{1}$ was calculated for asymmetric profiles. For seriously blended spectra of, e.g., binaries we selected single lines and calculated $v \sin i$ directly from the spectra.

Note again that no modeling of the data was undertaken for each star - we derive the value of differential rotation from comparing the ratio $q_{2} / q_{1}$ with results from models shown in Reiners \& Schmitt (2002a).

\section{Rotational velocities}

The zeros $\sigma_{n}$ of the Fourier transformed line profile from a rigidly rotating star with a linear limb darkening parameter $\epsilon$ scale with the projected rotational velocity $v \sin i$ (Dravins et al. 1990) as follows:

$\sigma_{n}=q_{n} / v \sin i$

$q_{1}=0.610+0.062 \epsilon+0.027 \epsilon^{2}+0.012 \epsilon^{3}+0.004 \epsilon^{4}$

$q_{2}=1.117+0.048 \epsilon+0.029 \epsilon^{2}+0.024 \epsilon^{3}+0.012 \epsilon^{4}$.

Thus we determine the value of $v \sin i$ from the zero positions $q_{2}$ and $q_{1}$ of the Fourier transformed broadening profile. Our values of $v \sin i$ are calculated for a limb darkening parameter $\epsilon=0.6$. For stars with a value of $v \sin i \lesssim 12 \mathrm{~km} \mathrm{~s}^{-1}$, the second zero $q_{2}$ cannot be measured since the signal vanishes in the noise at the relevant frequencies. In those cases $v \sin i$ was measured from the first zero only. For very low projected rotational velocities $\left(v \sin i \lesssim 3 \mathrm{~km} \mathrm{~s}^{-1}\right.$ ) even determining the first zero $q_{1}$ can be difficult and measurements must be interpreted as upper limits.

In the case of rigid rotation, the calculation of $v \sin i$ by $q_{1}$ and $q_{2}$ lead to the same values of $v \sin i$. As shown in Reiners \& Schmitt (2002a), for a star rotating differentially with the equator faster than the pole, $q_{1}$ becomes larger while $q_{2}$ becomes smaller. Thus, too small (large) a value of $v \sin i$ is calculated from $q_{1}\left(q_{2}\right)$ in the case of solar-like differential rotation. We took the mean of both results if both zeros were measured. Our simulations revealed that for a differentially rotating star this 


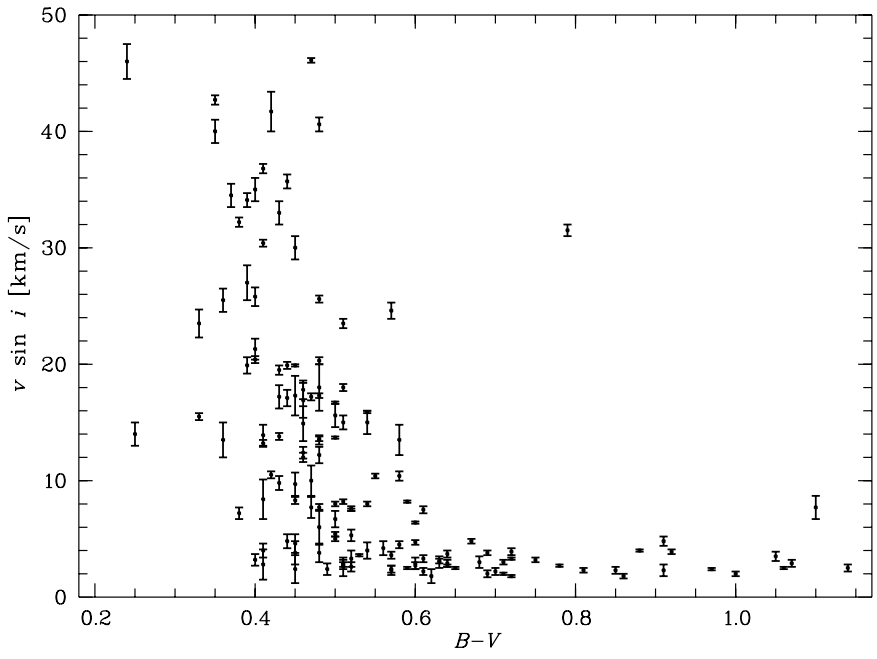

Fig. 5. Projected rotational velocities $v \sin i$ of the 142 stars of our primary sample. The exception from the slope consistent with magnetic braking at $B-V=0.79$ is the RSCVn HD 155555 .

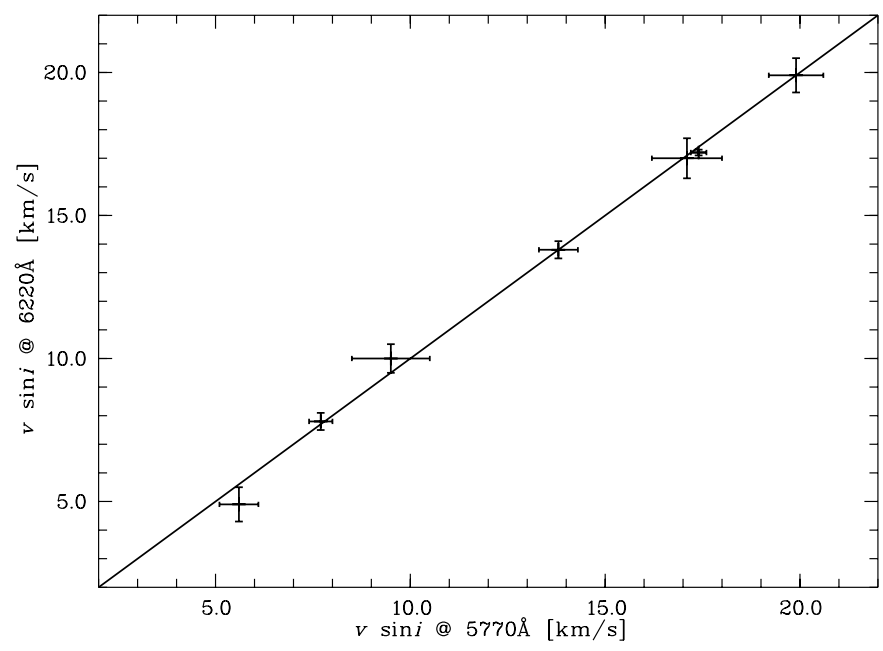

Fig. 6. Projected rotational velocities $v \sin i$ for the seven objects observed in the two regions between 5770-5810 ̊ and 6225-6270 ̊. Good agreement between the two regions can be seen. The value at $v \sin i=5 \mathrm{~km} \mathrm{~s}^{-1}$ is calculated only from $q_{1}$ and has a larger uncertainty.

is a good approximation for the projected rotational velocity at the equator. In those cases we take as error on $v \sin i$ the maximum deviation to the specific values of $v \sin i$ calculated from $q_{1}$ and $q_{2}$ alone.

Our values of the projected rotational velocities $v \sin i$ are given in Table A.1 together with the stars' names and colours from Hoffleit \& Warren (1991). If the measurements of $v \sin i$ are upper limits the values are marked with a " $<$ ". During our first observing run we also took a spectrum of the Sun by observing Jupiter's moon Ganymede. The spectrum was treated like the other spectra, and for the projected rotational velocity of the Sun we obtained a value of $v \sin i<2.1 \mathrm{~km} \mathrm{~s}^{-1}$, consistent with the actual value of $1.8 \mathrm{~km} \mathrm{~s}^{-1}$.

The derived values of $v \sin i$ for the stars of our primary sample are shown vs. $B-V$ colour in Fig. 5. A decline in $v \sin i$ consistent with rotational braking is apparent around

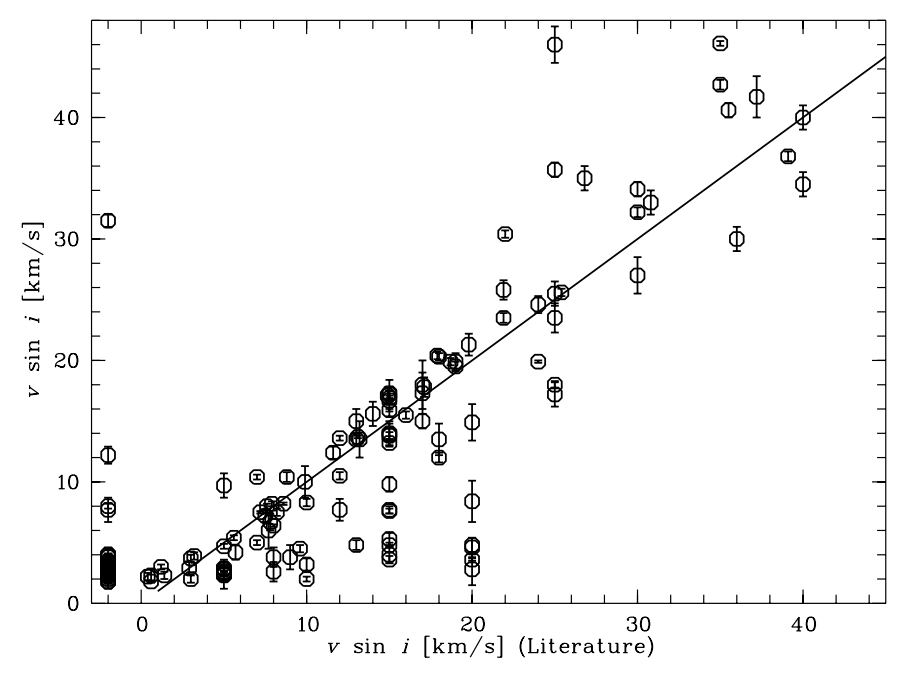

Fig. 7. Comparison of the calculated values of $v \sin i$ to the values in the literature (see text for references). Values of $v \sin i<20 \mathrm{~km} \mathrm{~s}^{-1}$ are often upper limits in the other works and thus appear under the 1:1 line drawn for comparison. The 30 stars without values in the references are plotted at $v \sin i_{\text {Lit. }}=-2 \mathrm{~km} \mathrm{~s}^{-1}$ for readability.

$B-V \approx 0.3$. The only exceptions at $B-V=0.79$ is member of an RSCVn system. For seven stars of our sample we have spectra in both wavelength regions between 5770-5810 $\AA$ and 6225-6270 $\AA$. A comparison of the derived values of $v \sin i$ is shown in Fig. 6; a good agreement within the error bars is obtained. A comparison to values of $v \sin i$ from the literature (Bernacca \& Perinotto 1970-1973; Fekel 1997; Groot et al. 1996; Saar \& Osten 1997; Uesugi \& Fukuda 1982) is plotted in Fig. 7. 30 of our sample stars were not mentioned in one of those catalogues and are plotted at $v \sin i_{\text {Lit. }}=-2 \mathrm{~km} \mathrm{~s}^{-1}$ for readability.

28 stars from the 30 , for which no rotational velocities were known to us, have values of $v \sin i$ lower than $10 \mathrm{~km} \mathrm{~s}^{-1}$ and are thus not suited for a differential rotation analysis with FTM. Additionally, most objects for which upper limits at about $10 \mathrm{~km} \mathrm{~s}^{-1}$ are cited in the literature turned out to have significantly lower values of $v \sin i$. In summary we have an overall number of 80 stars with $v \sin i<10 \mathrm{~km} \mathrm{~s}^{-1}$ in our sample. For these stars, no values of the differential rotation parameter $\alpha$ can be measured.

\section{Evidence for differential rotation}

Our data quality turned out to be adequate to push the noise down to a level sufficient to measure the second zero of the Fourier transform, $q_{2}$, on stars faster than $v \sin i=12 \mathrm{~km}^{-1}$. Four stars fall in the region $10 \mathrm{~km} \mathrm{~s}^{-1} \leq v \sin i<12 \mathrm{~km} \mathrm{~s}^{-1}$; 84 stars of our sample had too small a value of $v \sin i$ for a determination of differential rotation with FTM. Of the remaining stars five objects showed strong peculiarities (all five are binaries) and for them no mean profile could be derived with PLSD. 21 stars were sorted out due to asymmetries found after mirroring. Thus the ratio $q_{2} / q_{1}$ could be determined in 32 of the investigated 142 stars.

The 26 stars rotating faster than $v \sin i=12 \mathrm{~km}^{-1}$ but not suited for FTM are given in Table 2. In column four we indicate 
Table 2. Stars with peculiar or asymmetric profiles.

\begin{tabular}{rcccl}
\hline \hline HD & $M_{V}^{a}$ & $T_{\text {eff }}^{a}$ & PLSD & Comment \\
\hline 2726 & 2.57 & 7028 & & extremely weak lines, SB? \\
3302 & 3.01 & 6463 & $\sqrt{ }$ & \\
8556 & 3.27 & 6565 & $\sqrt{ }$ & \\
25570 & 2.68 & 6686 & $\sqrt{ }$ & \\
40136 & 2.88 & 6943 & $\sqrt{ }$ & \\
57749 & 0.18 & 6875 & & SB \\
58728 & 3.12 & 6451 & & SB (with different $v$ sin $i$ 's) \\
98991 & 2.37 & 6463 & $\sqrt{ }$ & \\
99285 & 2.04 & 6568 & $\sqrt{ }$ & \\
104827 & 1.82 & 7535 & $\sqrt{ }$ & center \\
110379 & 3.54 & 7683 & $\sqrt{ }$ & flank \\
113848 & 2.82 & 6581 & $\sqrt{ }$ & center \\
114642 & 2.69 & 6255 & $\sqrt{ }$ & \\
118646 & 2.58 & 6618 & $\sqrt{ }$ & center \\
123999 & 2.99 & 6118 & & SB \\
128898 & 3.12 & 7847 & $\sqrt{ }$ & \\
144069 & & & $\sqrt{ }$ & flank \\
153363 & 3.25 & 6697 & $\sqrt{ }$ & \\
155555 & 3.89 & & & fast RSCVn \\
156897 & 3.11 & 6645 & $\sqrt{ }$ & \\
160032 & 2.98 & 6500 & $\sqrt{ }$ & flank \\
160910 & 2.66 & 6615 & $\sqrt{ }$ & \\
176303 & 2.59 & 5959 & $\sqrt{ }$ & \\
186185 & 2.57 & 6390 & $\sqrt{ }$ & center \\
199684 & 2.96 & 6695 & $\sqrt{ }$ & \\
220729 & 3.01 & 6699 & $\sqrt{ }$ & \\
\hline & & & &
\end{tabular}

a Calculated from $u v b y \beta$ photometry (see Sect. 7), data from Hauck \& Mermilliod (1998).

if a mean profile was derived with PLSD, in column six we give a comment on the asymmetry. The comment "center" indicates that the mean profile has an asymmetric line core while the flanks appear symmetric, similar to the case shown in Fig. 4a, "flank" indicates that the flanks on the blue and the red side differ, similar to the case shown in Fig. 4b. If no comment is given, the situation is similar to Fig. $3 b$, i.e., no specific signature of asymmetry is apparent.

Following Reiners \& Schmitt (2002a), a ratio $q_{2} / q_{1}<1.72$ is a direct indication for solar-like differential rotation (equator faster than pole). $1.72<q_{2} / q_{1}<1.83$ is typical for solid rotation with an arbitrary limb darkening parameter $\epsilon$. A value of $q_{2} / q_{1}>1.83$ indicates anti solar-like differential rotation (pole faster than rotator) or a polar spot (Reiners \& Schmitt 2002b).

The measured values of $q_{2} / q_{1}$ of the 32 stars are given in column seven of Table 3 . In Fig. 8 the distribution of the measurements of $q_{2} / q_{1}$ among the 32 profiles is shown. The region consistent with rigid rotation of an unspotted star is indicated by dotted lines. The majority of our objects ( 20 of 32) lie within that region. Ten of the 32 objects show significant differential rotation with $q_{2} / q_{1}<1.72$, only two objects have $q_{2} / q_{1}>1.83$.

Four stars of the 32 were observed in both wavelength regions between 5770-5810 А and 6225-6270 A. The derived values of $q_{2} / q_{1}$ are compared in Fig. 9. For all four stars the derived values are compatible within the observational errors.

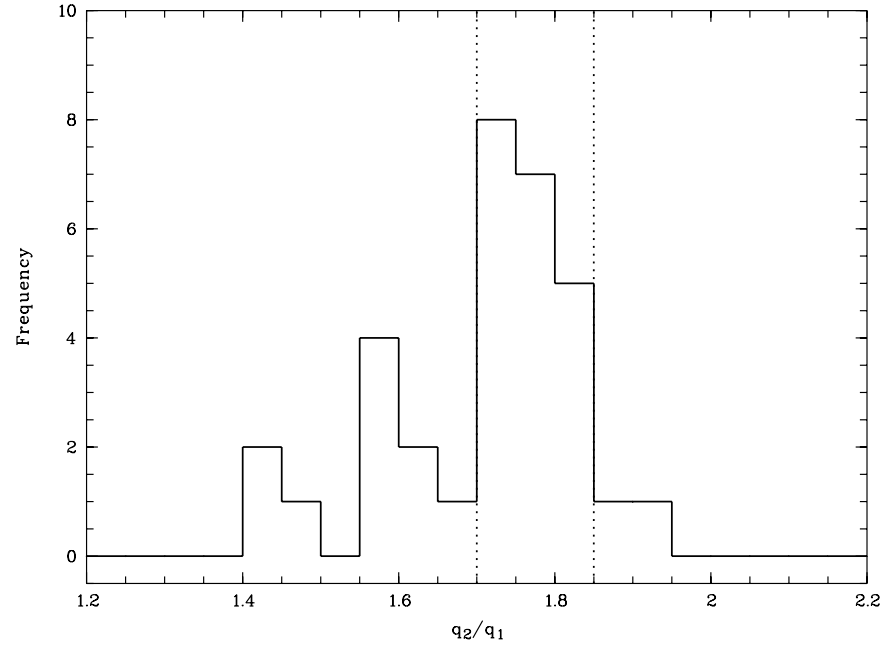

Fig. 8. Distribution of the ratio $q_{2} / q_{1}$ among the 32 analyzed profiles. The region between the dotted lines is occupied by solid rotators with arbitrary limb darkening coefficient according to a linear limb darkening law. $q_{2} / q_{1}<1.72$ indicates solar-like differential rotation, $q_{2} / q_{1}>1.83$ can be due to anti solar-like differential rotation or a polar spot.

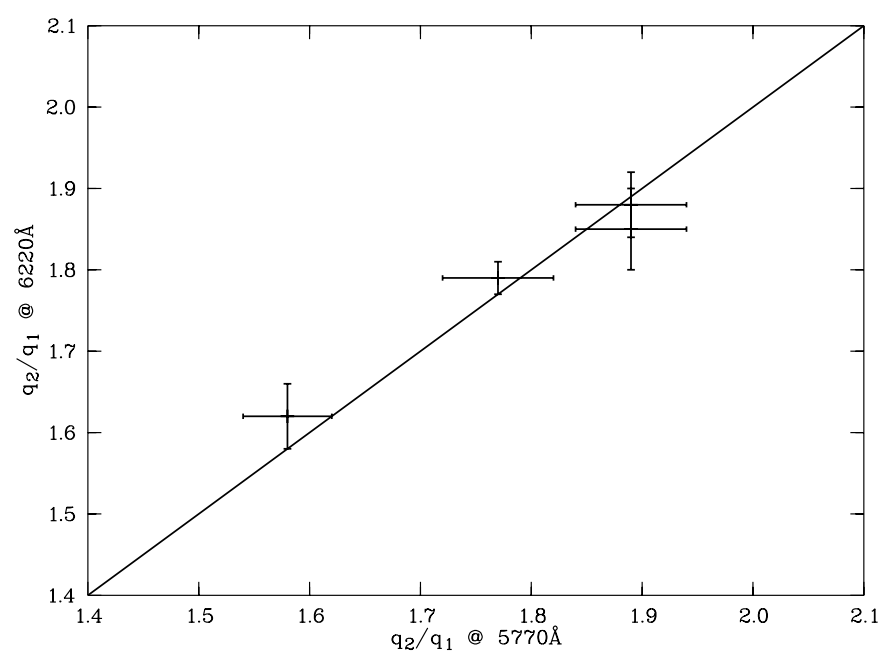

Fig. 9. Comparison of the measured values of $q_{2} / q_{1}$ for the four stars observed in two different wavelength regions between $5770-5810 \AA$ and 6225-6270 $\AA$ for that $q_{2} / q_{1}$ could be measured. Within the observational errors the results from different wavelength regions are consistent with each other.

Although both wavelength regions are relatively close to each other, this results demonstrates that the derived values of $q_{2} / q_{1}$ are not due to an effect specific for the utilized absorption lines but to an underlying universal line broadening.

For three stars, HD 89449, HD 121370 and HD 173667 , we find extremely low values of $q_{2} / q_{1}$, and the strong deviation to the profiles expected from a rigid rotator can easily be seen even in the data domain. In Fig. 10 the profiles are shown in Fourier and data domain; in the left panels of Fig. 10 the Fourier transform of the broadening profiles derived with PLSD are shown with their error bars. Note that the second zero $q_{2}$ is visible in all cases. With dashed lines we overplotted the Fourier transform of a rigid rotator with the same value of $v \sin i, \epsilon=0.6$ and a radial-tangential macroturbulence of 

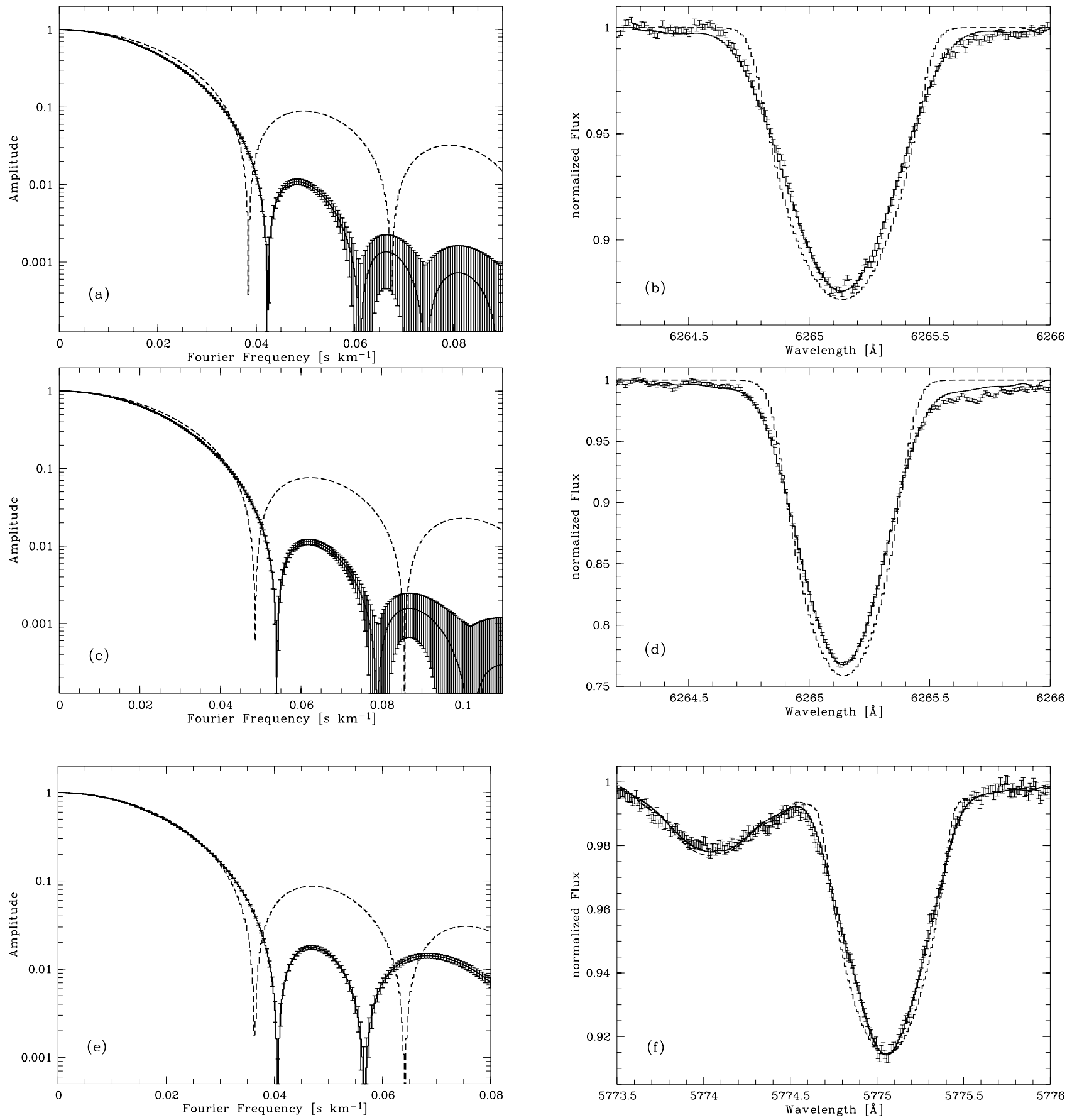

Fig. 10. Fourier spectra and small wavelength regions of the three stars with $q_{2} / q_{1}<1.5$. Left column: Fourier transforms of the broadening profiles derived with PLSD and their error bars. Right column: the original data plotted as error bars, overplotted with the results of the convolution of the appropriate template with the overall broadening profile (solid line, the Fourier transform of that profile is given in the left column). a) and b): $\left.\operatorname{HD} 89449, q_{2} / q_{1}=1.44 \pm 0.04 ; \mathbf{c}\right)$ and d): $\left.\operatorname{HD} 121370, q_{2} / q_{1}=1.46 \pm 0.03 ; \mathbf{e}\right)$ and f): $\mathrm{HD} 173667, q_{2} / q_{1}=1.40 \pm 0.02$. For comparison broadening profiles of rigid rotators are overplotted with dashed lines. The same values of $v \sin i$ as obtained for the individual stars are chosen, radial-rangential turbulence velocity of $v_{\mathrm{RT}}=4 \mathrm{~km} \mathrm{~s}^{-1}$ and a linear limb darkening parameter of $\epsilon=0.6$ are applied. Note that no optimization of the rigidly rotating case with, e.g., turbulence or limb darkening has been attempted (see text).

$v_{\mathrm{RT}}=4 \mathrm{~km} \mathrm{~s}^{-1}$. Note that we did not optimize the profile of the rigidly rotating case, nor did we determine values of turbulent velocities for the differential case. The amplitude of the Fourier transform depends on a variety of parameters and all photospheric velocities influence it. We think that it is always possible to find suitable values that produce the general slope of the Fourier transform. Differences in amplitude do not indicate bad fit quality.

On the other hand, the zeroes of a Fourier transform and especially the ratio $q_{2} / q_{1}$ are not affected by the "common" velocity fields and also limb darkening has only a limited effect (cf. Reiners \& Schmitt 2002a). Differential rotation is the only 


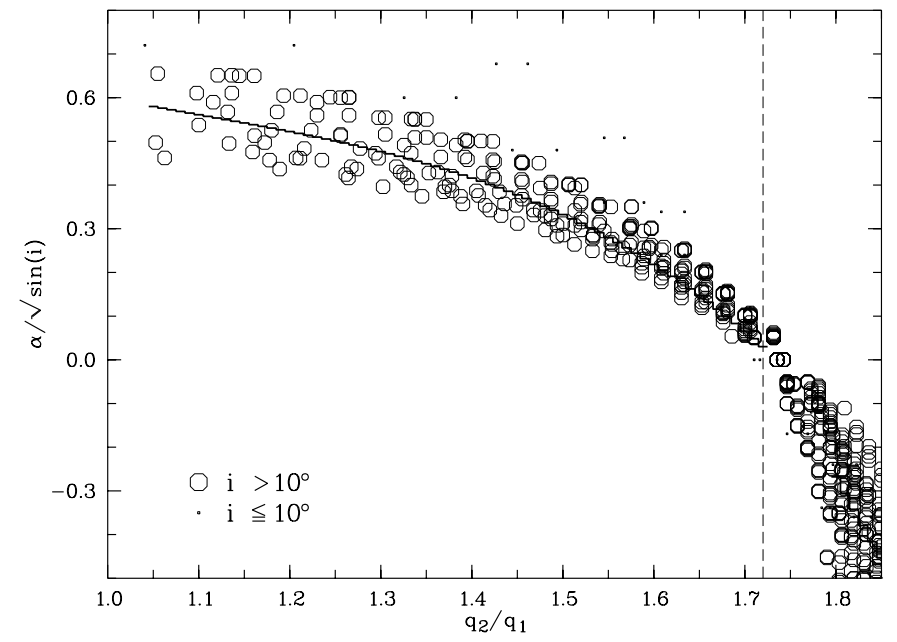

Fig. 11. Data points from our simulations; each point represents a calculated model. Differential rotation $\alpha$ and inclination angle $i$ are given, and the ratio $q_{2} / q_{1}$ is measured from the simulated spectrum. For inclination angles $i>10^{\circ}$ the ratio $\alpha / \sqrt{\sin i}$ depends monotonically on $q_{2} / q_{1}$. The solid line represents Eq. (5), the dashed line is at $q_{2} / q_{1}=1.72$, the smallest ratio consistent with rigid rotation.

known mechanism that can cause ratios of $q_{2} / q_{1}<1.72$. Such small ratios are apparent in the three left plots of Fig. 10 and are in obvious contrast to the ratios of the rigid cases.

In the right column of Fig. 10 we plot the same cases in the data domain. Note that the data, not the derived broadening profiles are shown directly. Over the error bars of the data we plotted as a solid line the convolutions of our derived templates with the derived broadening profiles. The consistency of the result of the convolution and the data is remarkable.

Even with the error bars of the spectra, that are quite large compared to those of the broadening profiles derived from the complete region of $\sim 40 \AA$, the deviations of the rigidly rotating cases from the data are obvious. Again, it is not the incompatibilty in the wings or in the core that rules out rigid rotation, it is the overall shape of the profiles and especially the steepness of the flanks on both sides of the line. Such shapes cannot be induced by strong limb darkening $\epsilon$ or large turbulent velocities. In Figs. 10b,d,f the effects of differential rotation are directly evident in the spectra.

\section{Deriving $\alpha$}

A more physical parameter to describe a star's differential rotation is the relative shear $\alpha=\Delta \Omega / \Omega_{0}$. Given the rotational period we can thus also obtain $\Delta \Omega$ from $\alpha$, a value often assumed to be constant for differentially rotating stars with different rotational velocities. We already noted that a measured ratio of $q_{2} / q_{1}<1.72$ indicates differential rotation with the equator faster than the pole. In this section we show a direct connection between $\alpha$ and $q_{2} / q_{1}$.

In our simulations of line profiles from differentially rotating stars we calculated the ratios $q_{2} / q_{1}$ determined from the spectral lines of stars with given $\alpha$ in the range $-0.5 \leq \alpha \leq 1.0$ and $v \sin i=10 \mathrm{~km} \mathrm{~s}^{-1}$ observed under an inclination angle $i$ in the range $5^{\circ} \leq i \leq 90^{\circ}$ in steps of $5^{\circ}$. As shown in Fig. 6 of
Reiners \& Schmitt (2002a), the ratio $q_{2} / q_{1}$ depends nonlinearly on both $\alpha$ and $i$, thus it is only possible to derive a combined value of $\alpha$ and $i$ from an observed ratio $q_{2} / q_{1}$.

In Fig. 11 we plot the ratio $\alpha / \sqrt{\sin i}$ vs. $q_{2} / q_{1}$ obtained from our simulations. Open circles mark data points with an inclination $i>10^{\circ}$, dots represent values from stars seen under an inclination angle $i$ of $5^{\circ}$ or $10^{\circ}$. Obviously, the ratio $\alpha / \sqrt{\sin i}$ is a good description of what is contained in the observable $q_{2} / q_{1}$ for inclination angles $i>10^{\circ}$. The probability to observe a star under an inlination $i \leq 10^{\circ}$ is $\sim 1.5 \%$, on the other hand, for $i=10^{\circ}$ the projected rotational velocity becomes $0.17 v$ and stars with $v \sin i>10 \mathrm{~km} \mathrm{~s}^{-1}$ must have equatorial velocities of $v \gtrsim 60 \mathrm{~km} \mathrm{~s}^{-1}$. Therefore, in our sample of 32 stars we do not expect to have more than one of those cases and neglect possible inclinations $i \leq 10^{\circ}$ in the following.

Again, ratios of $1.72<q_{2} / q_{1}<1.83$ are consistent with rigid rotation while $q_{2} / q_{1}<1.72$ indicates solar like differential rotation; the dashed line in Fig. 11 marks $q_{2} / q_{1}=1.72$. In the region $q_{2} / q_{1}<1.72$ the dependence of $\alpha / \sqrt{\sin i}$ on $q_{2} / q_{1}$ can be approximated by the polynomial

$$
\frac{\alpha}{\sqrt{\sin i}}=2.74-5.16 x+4.32 x^{2}-1.30 x^{3}, \quad x=\frac{q_{2}}{q_{1}} .
$$

Equation (5) is shown with a solid line in Fig. 11. Maximum deviations around Eq. (5) are of the order of $\Delta(\alpha / \sqrt{\sin i})=0.1$ at $q_{2} / q_{1}=1.2$ and $\Delta(\alpha / \sqrt{\sin i})=0.05$ at $q_{2} / q_{1}=1.65$. The values of $\alpha / \sqrt{\sin i}$ derived from Eq. (5) are shown in column nine of Table 3 . The uncertainty in $\alpha / \sqrt{\sin i}$ given in column ten of Table 3 is the combined error from the uncertainty $\delta q_{2} / q_{1}$ and the scatter in the $\alpha / \sqrt{\sin i}\left(q_{2} / q_{1}\right)$-relation in Eq. (5).

Ratios of $q_{2} / q_{1}>1.83$ can be due to anti-solar like differential rotation but are more likely due to a polar spot (cf. Reiners \& Schmitt 2002b), thus we do not propose differential rotation for them. In the region $q_{2} / q_{1}>1.83$ the simulated data points can be fitted by

$$
\frac{\alpha}{\sqrt{\sin i}}=6.41-3.71 x, \quad x=\frac{q_{2}}{q_{1}}
$$

For $q_{2} / q_{1}>1.72$ we assign $\alpha=0.0$ for the reasons mentioned above but give the largest amount of differential rotation consistent with our measurements as uncertainty in column ten of Table 3. Negative values of $\Delta \alpha / \sqrt{\sin i}$ indicate that only anti-solar like differential rotation is - besides a polar spot consistent with the measured value of $q_{2} / q_{1}$.

\section{Results}

The obtained values $q_{2} / q_{1}$ and $\alpha / \sqrt{\sin i}$ for the 32 stars, as well as parameters from the literature are presented in Table 3 . Ten stars with significant evidence for solar-like differential rotation have been found.

$B-V$ colours are taken from the Bright Star Catalogue (Hoffleit \& Warren 1991). Values $M_{V}$ and $T_{\text {eff }}$ are calculated from $u v b y \beta$ photometry using the program $U V B Y$ BETA published by Moon (1985); data are from Hauck \& Mermilliod (1998). For $T_{\text {eff }}$ a new calibration by Napiwotzki et al. (1993) based on the grids of Moon \& Dworetsky (1985) was used, the statistical error of the temperature determination is about 

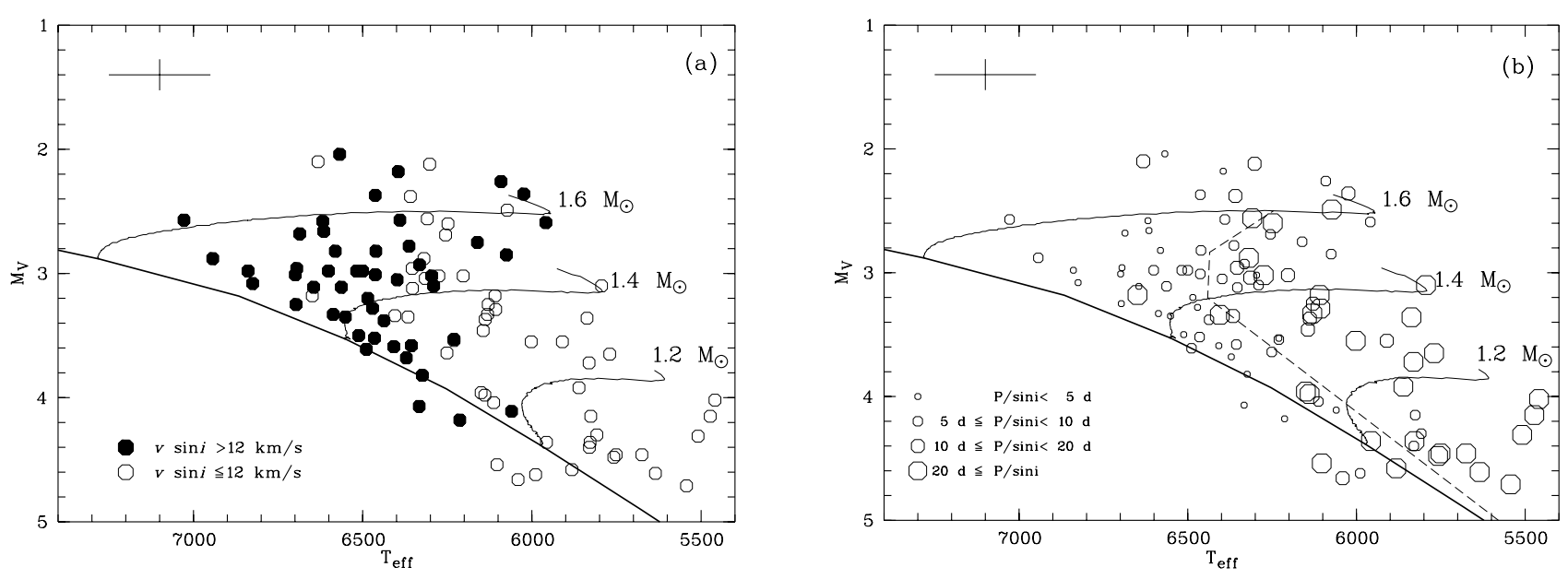

Fig. 12. HR-diagram of our sample stars. Absolute visual magnitudes $M_{V}$ and effective temperatures $T_{\text {eff }}$ are calculated from $u v b y \beta$-photometry (see text). Typical error bars are given in the upper left corner, the zero age main sequence (ZAMS) and evolutionary tracks for 1.2, 1.4 and 1.6 $M_{\odot}$ from Siess et al. (2000) are overplotted. a) Stars with $v \sin i>12 \mathrm{~km} \mathrm{~s}^{-1}$ are plotted with full circles, slower rotators with open circles. b) Rotational periods; larger symbols indicate longer rotational periods.

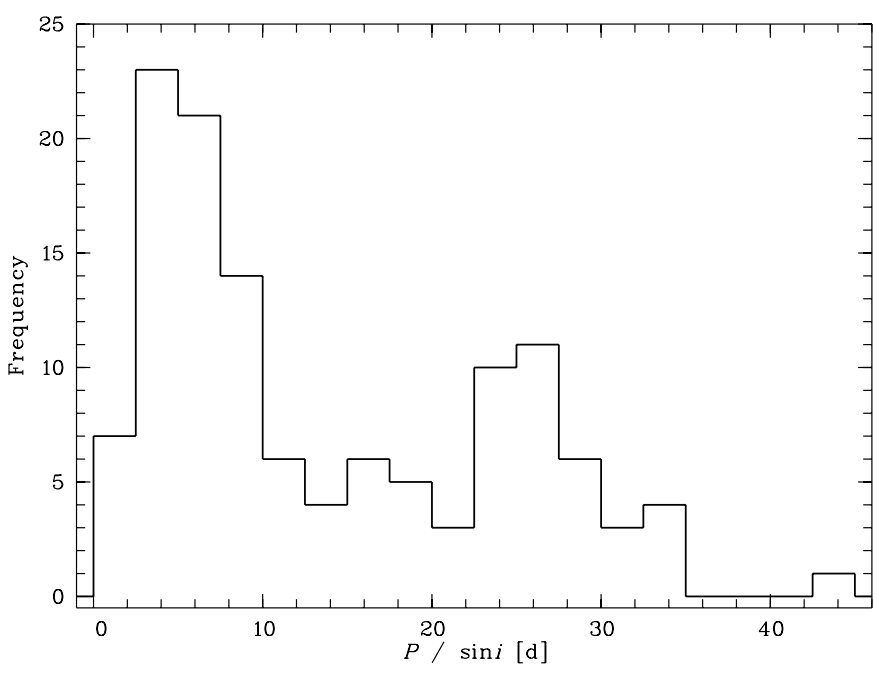

Fig. 13. Distribution of rotational periods $P / \sin i$ derived from the projected rotational velocities $v \sin i$ and stellar radii in our primary sample.

$\Delta T_{\text {eff }}=150 \mathrm{~K}$. Distances are derived from the Hipparcos parallaxes (ESA 1997). Sources for lithium abundances $\log \epsilon(\mathrm{Li})$ are given in Table 3, upper boundaries of $\log \epsilon(\mathrm{Li})$ are marked. The values of $\mathrm{Ca}$ II $\mathrm{H}$ and $\mathrm{K}$ emission $\log R_{\mathrm{HK}}^{\prime}$ are calculated from the parameter $\langle S>$ from Baliunas et al. (1995) using the calibration given in Noyes et al. (1984). X-ray data are taken from Hünsch et al. (1998, Hünsch et al. 1999) except for HD 89449.

To normalize the X-ray luminosity $L_{X}$ to the bolometric luminosity $L_{\mathrm{bol}}$, the latter is calculated from $M_{\mathrm{V}}$ using the bolometric correction B.C. given in Reed (1998), that is an empirical fit to the data given in Lang (1992). The bolometric correction was also used for the stellar radii; $R$ is calculated from $M_{\text {bol }}$ and $T_{\text {eff }}$ with the calibration given in Cox (2000).

\subsection{Rotational velocities and periods in the HR-diagram}

All (presumably single) stars of our primary sample are plotted in an HR-diagram in Fig. 12a. Stars with values of $v \sin i>$ $12 \mathrm{~km} \mathrm{~s}^{-1}$ are shown with full circles, stars with $v \sin i \leq$ $12 \mathrm{~km} \mathrm{~s}^{-1}$ with open circles. The zero age main sequence (ZAMS) and evolutionary tracks for 1.2, 1.4 and $1.6 M_{\odot}$ from Siess et al. (2000) are overplotted; the models with solar composition including overshooting were chosen. A drop of rotational velocity is apparent somewhere around spectral type F7 or $6200 \mathrm{~K}$. This drop is well documented in many works (for a summary, cf. Gray 1988). As mentioned above, for measuring differential rotation data quality limits us to stars with projected rotational velocities larger than $12 \mathrm{~km} \mathrm{~s}^{-1}$, that is in our sample to stars of spectral type earlier than $\mathrm{G} 1$.

We derived rotational periods $P / \sin i$ for our sample stars from the measured values of $v \sin i$ and radii derived from $u v b y \beta$-photometry. The different periods are plotted using different symbol sizes in the HR-D in Fig. 12b; larger symbols indicate longer rotational periods. The distribution of $P / \sin i$ in our sample is shown in Fig. 13. Some indication for a bimodal period distribution may be found. In Fig. 12b we overplotted the isochrone at 2 Gyr according to Siess et al. (2000). Dividing our sample into groups of stars younger and older than $2 \mathrm{Gyr}$ we find that 22 out of 52 "young" stars have rotational periods of $P / \sin i<5 \mathrm{~d}(42 \%)$, while only 3 out of 49 "old" stars (6\%) fall into this group. Thus we find that a correlation between age in the HR-D and the projected rotational period $P / \sin i-$ as expected from rotational braking - is significant on a $4 \sigma$-level.

\subsection{Profile symmetry of the fast rotators}

In Fig. 14 we plot in an HR-diagram the 32 objects for that differential rotation was derived (filled circles) together with the stars that were discarded due to asymmetries in the spectral lines (open circles). The stars with asymmetric profiles tend to have higher masses and populate the upper left region of 


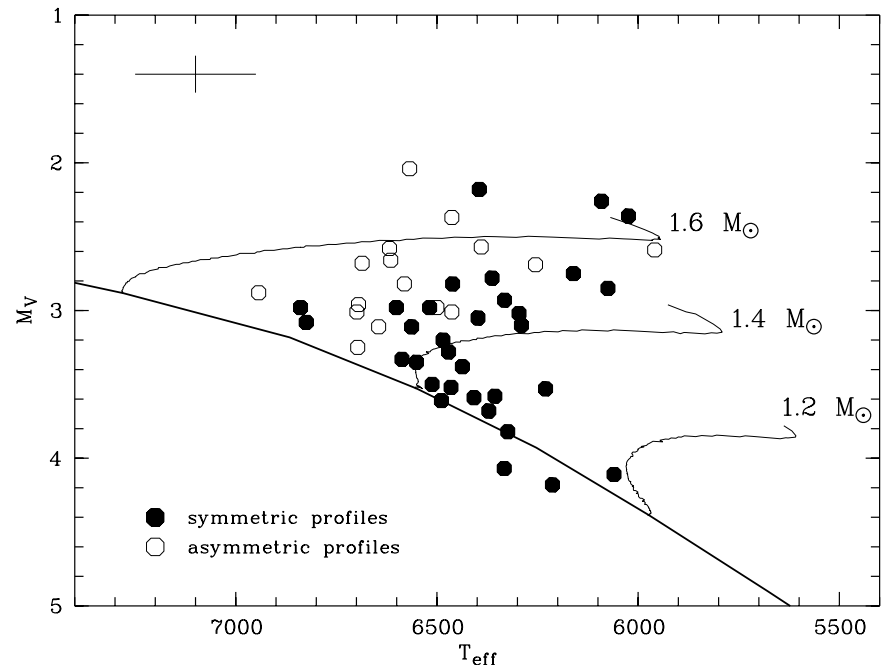

Fig. 14. HR-diagram as in Fig. 12. Only stars with $v \sin i>12 \mathrm{~km} \mathrm{~s}^{-1}$ are plotted; stars with symmetric profiles as full circles, those with asymmetric profiles with open circles.

the HR-D, while the stars with symmetric profiles have lower masses and lie in the lower right part of the HR-D. The region populated by the stars with asymmetric profiles is directly neighboured to the "granulation boundary" found by Gray (1991). He found that stars above that boundary show asymmetries in the profiles an order of magnitude larger than those found in spectral lines of stars below the boundary. Thus it is not surprising that many single stars in that region show asymmetric profiles. Although the reason for the onset of larger asymmetries in that region is not well known, it appears that the 21 discarded stars are not randomly distributed in the HR-D, but that the profile asymmetries seem to be of physical origin.

The 32 stars with symmetric broadening profiles are the objects we can examine in our search for differential rotation. This subsample consists of stars of spectral type F0 - G0 and effective temperatures between 6000 and $6900 \mathrm{~K}$. From the evolutionary tracks in Fig. 14 we find that their masses cluster between 1.2 and $1.6 M_{\odot}$ and that stars of widely varying ages are in the sample.

Of the 21 stars with asymmetric broadening profiles, twelve (60\%) have values of $v \sin i>20 \mathrm{~km} \mathrm{~s}^{-1}$, while that is only the case for 11 of the 32 stars $(34 \%)$ with symmetric profiles. While this is not surprising since the rotational velocities of stars of earlier spectral types tend to have higher rotational velocities, we remark that sorting out stars with asymmetric profiles for the most part neglects the faster rotators. Thus a statistical interpretation of the velocity dependence of stellar parameters has to be carried out with great care.

\subsection{Rotation and $X$-ray emission}

The X-ray luminosities $L_{\mathrm{X}}$ observed with the $R O S A T$ satellite are plotted against the values of $v \sin i$ for our sample stars in Fig. 15. Different spectral types are indicated by different symbols, the stars with evidence for solar-like differential rotation are plotted with filled symbols. In agreement with Pallavicini et al. (1981), F-stars show only a weak correlation between

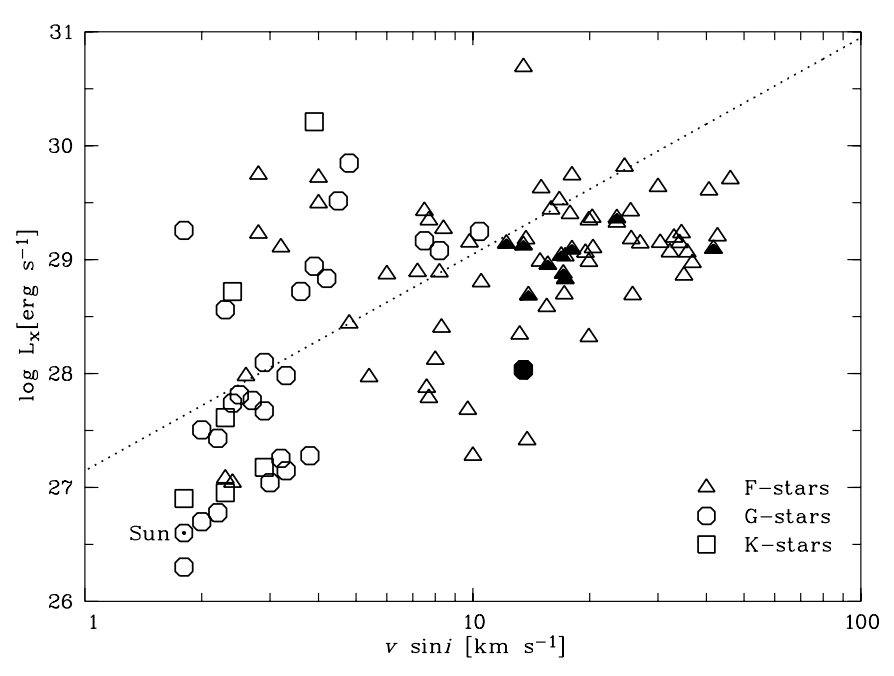

Fig. 15. X-ray luminosities $L_{\mathrm{X}}$ from $\operatorname{ROS} A T$ vs. our values of $v \sin i$ for all stars of our sample. Different spectral types are indicated by different symbols, filled symbols represent stars with evidence for solar like differential rotation. The least squares fit to Einstein data of stars from F7 to M5, given in Pallavicini et al. (1981), is overplotted.

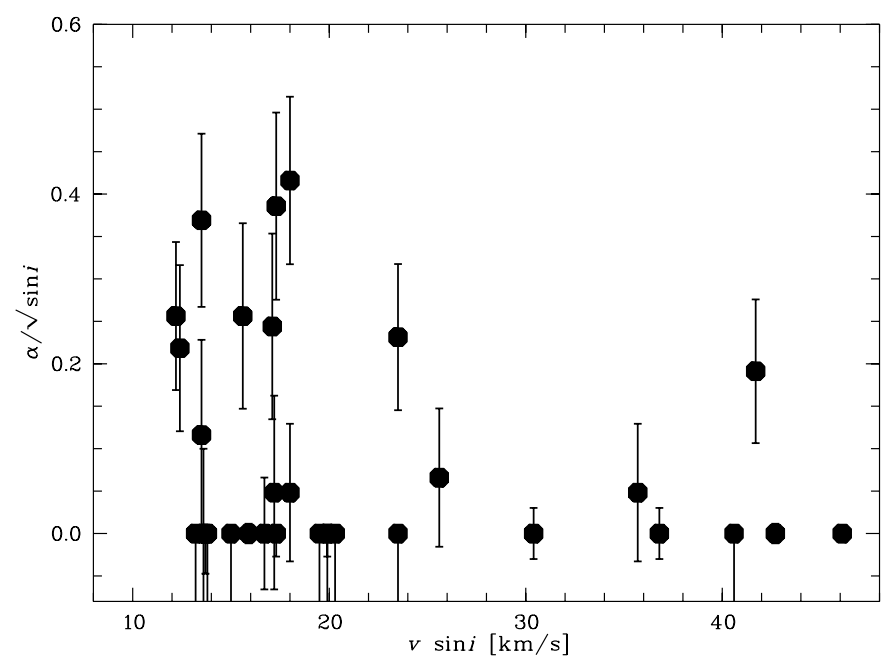

Fig. 16. Derived values of $\alpha / \sqrt{\sin i}$ vs. $v \sin i$ for the 32 of our sample stars where measurement of differential rotation was possible. Stars for that a ratio of $q_{2} / q_{1}$ is measured are interpreted as rigid rotators $(\alpha=0.0)$, possible anti-solar like differential rotation $(\alpha<0.0)$ is indicated by the error bars.

X-ray luminosity and equatorial rotational velocity. Our sample of later spectral type stars, however, does not follow the correlation derived there. We overplotted their correlation between $L_{\mathrm{X}}$ and $v \sin i$ as dotted line in Fig. 15. Although our data do not necessarily contradict their correlation, the scatter among the stars later than G0 is substantially higher than in Fig. 5 of Pallavicini et al. (1981). Nevertheless, we confirm the existence of a velocity dependent lower envelope of X-ray luminosities at about an order of magniude lower than described by the correlation given in Pallavicini et al. (1981). 

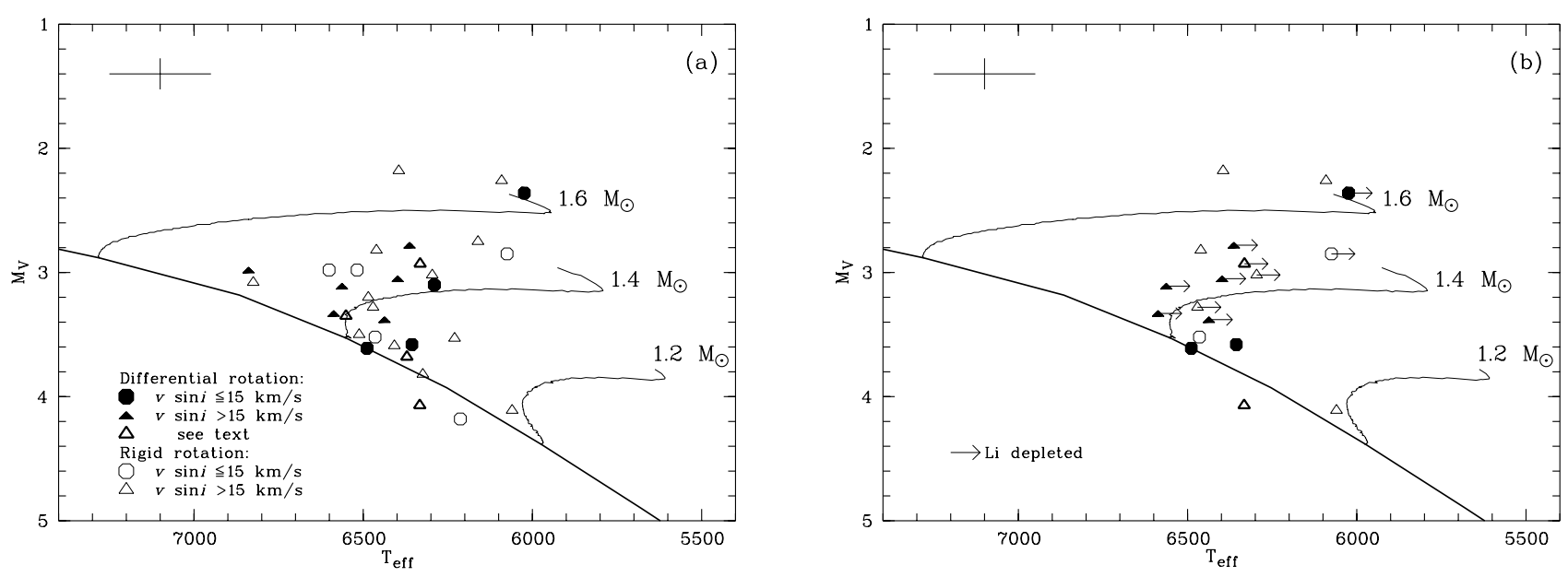

Fig. 17. HR-diagram of a) the 32 stars with measured values of $q_{2} / q_{1}$. Slow rotation $\left(v \sin i<15 \mathrm{~km} \mathrm{~s}^{-1}\right)$ is indicated by circles, fast rotation by triangles. Full and open symbols represent stars with and without significant evidence for differential rotation, respectively. Four fast rotators with measured values of $\alpha / \sqrt{\sin i}>0.0$ but error bars consistent with rigid rotation are symbolized with bold triangles; b) same as a) but only objects with available data on $\mathrm{Li}$ abundances are shown. "Li-depletion" $(\log \epsilon(\mathrm{Li}) \leq 2.2)$ is indicated by a right arrow while all other stars have $\log \epsilon(\mathrm{Li})>2.2$.

\subsection{Differential rotation and $v \sin i$}

In Fig. 16 we plot the amount of differential rotation $\alpha / \sqrt{\sin i}$ vs. the projected rotational velocity $v \sin i$ of the 32 stars where a measurement of differential rotation was possible. As pointed out in Sect. 6, rigid rotation is proposed for the stars with ratios of $q_{2} / q_{1} \geq 1.72$. However, since anti-solar like differential rotation is consistent with larger ratios of $q_{2} / q_{1}$, some of the objects have large error bars in the direction of negative $\alpha$. Stronger differential rotation appears to be more common in the slowly rotating stars $(41 \%$ of the rigid rotators have values of $v \sin i>20 \mathrm{~km} \mathrm{~s}^{-1}$ while that is the case for only $20 \%$ of the differentially rotating stars), but we might have sorted out specifically the differentially rotating stars while neglecting the stars with asymmetric broadening profiles. Evidence for differential rotation is also found on stars with higher rotational velocities. We find no upper boundary in $v \sin i$ for the onset of differential rotation.

In Fig. 17a we show the 32 stars in an HR-D with symbols characterizing their rotational velocity and differential rotation. Triangles represent stars with $v \sin i>15 \mathrm{~km} \mathrm{~s}^{-1}$, circles represent slower ones. Filled symbols stand for differential rotators while rigid rotators are shown with open symbols. In four cases we find positive values of $\alpha / \sqrt{\sin i}$ but with errors larger than the actual values of $\alpha / \sqrt{\sin i}$. These objects are marked with bold triangles to indicate that rigid rotation cannot be excluded for these stars.

Differential rotators are distributed over the whole region populated by our sample stars and no preferred region is apparent either for differential or for solid rotators. No correlation between (i) differential rotation and mass, and (ii) differential rotation and age is found.

\subsection{Li abundances}

The correlation between differential rotation and $\mathrm{Li}$ abundances was studied by Reiners \& Schmitt (2002d). They showed that for stars with projected rotational velocities $v \sin i>15 \mathrm{~km} \mathrm{~s}^{-1}$ a correlation between $\mathrm{Li}$ abundace and evidence for differential rotation exists. Specifically, among the fast rotators ( $v \sin i>15 \mathrm{~km} \mathrm{~s}^{-1}$ ), the differentially rotating stars tend to be Li-depleted, while a large spread in $\mathrm{Li}$ abundance can be found among the rigidly rotating stars. The $\mathrm{Li}$ abundances of slow rotators are generally more spread out and differential rotation as well as Li-depletion seem to be more common in slowly rotating stars.

In Fig. 17b the stars with measured differential rotation, for which Li abundances are available, are plotted in the same way as in Fig. 17a. Here we interpret stars for which no Li was detected and only an upper limit exists as Li-depleted, these objects are marked with a right arrow in Fig 17b. For objects without right arrows $\mathrm{Li}$ was detected, i.e., $\log \epsilon(\mathrm{Li})>2.2$ in our sample. In Fig. 17b it is obvious that fast differentially rotating Li-depleted stars are not specifically evolved. Even some fast rigid rotators are at least of comparable age if not older. We think that Li-depletion in these relatively young stars is presumably a consequence of mixing processes connected with differential rotation (cf. Balachandran 1990).

\subsection{Differential rotation and activity}

In Fig. 18 we plot the dependence of differential rotation on the two most important indicators of stellar activity; Fig. 18a shows the value of $\alpha / \sqrt{\sin i}$ vs. the chromospheric emission from Ca II $\mathrm{H}$ and $\mathrm{K}$ lines in terms of $\log R_{\mathrm{HK}}^{\prime}$ (cf. Noyes et al. 1984), Fig. 18b shows $\alpha / \sqrt{\sin i}$ vs. the X-ray luminosity normalized to the bolometric luminosity $\log L_{\mathrm{X}} / L_{\mathrm{bol}}$. In both figures we indicate available measurements of the $\mathrm{Li}$ abundance with different symbols. Full circles represent stars where Li measurements provide only upper limits; these stars are Li-depleted. Open circles stand for "Li rich" stars and an asterisk indicates that no Li measurement is available to our knowledge. 

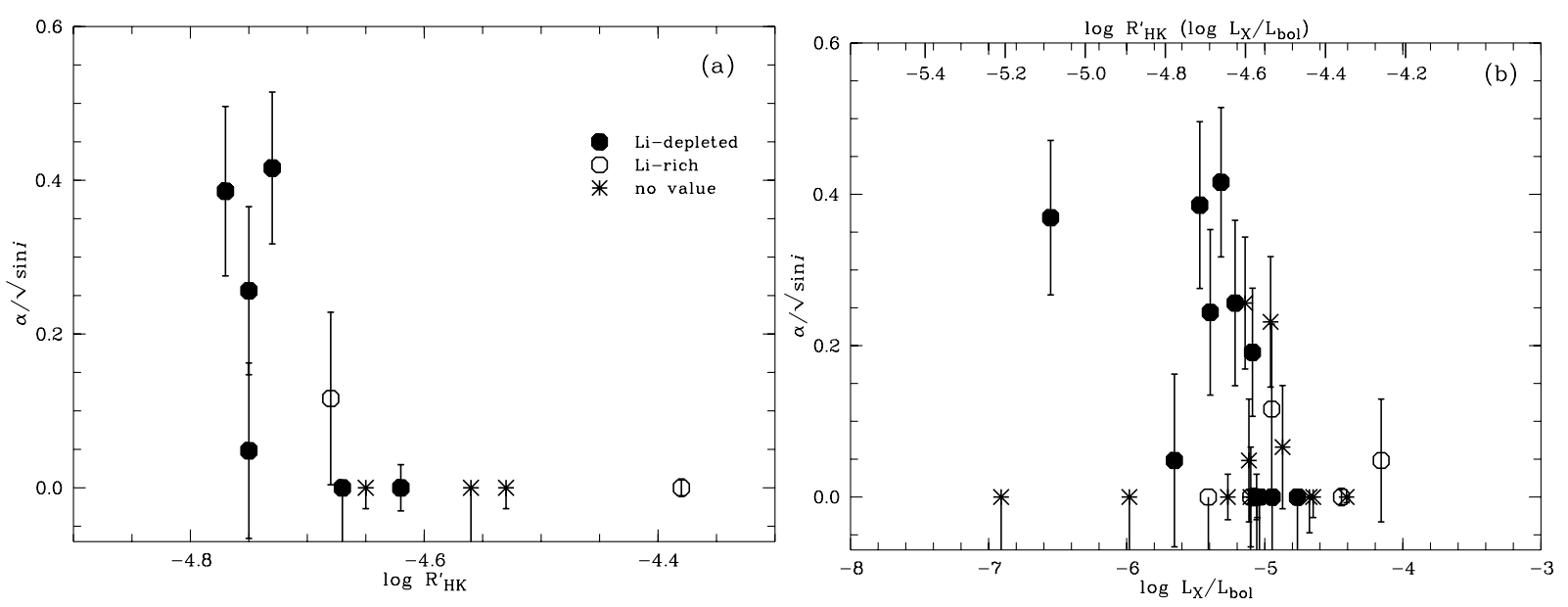

Fig. 18. Differential rotation in terms of $\alpha / \sqrt{\sin i}$ vs. activity indicators. a) Chromospheric Ca II $\mathrm{H}$ and $\mathrm{K}$ emission log $R_{\mathrm{HK}}^{\prime}$; b) X-ray luminosity $L_{\mathrm{X}}$ normalized to bolometric luminosity $L_{\mathrm{bol}}$. Available values of $\mathrm{Li}$ abundances are indicated with symbols. At the top panel of (b), $\log R_{\mathrm{HK}}^{\prime}$ calculated from $\log L_{\mathrm{X}} / L_{\mathrm{bol}}$ according to the calibration of Sterzik \& Schmitt (1997) is marked.

In Fig. 18a a trend is apparent; while no differential rotation is found for stars with a value of $\log R_{\mathrm{HK}}^{\prime}>-4.65$, $\alpha / \sqrt{\sin i}>0$ is consistent with our results for all stars in the region $-4.8<\log R_{\mathrm{HK}}^{\prime}<-4.65$. No measurements for stars less active that $\log R_{\mathrm{HK}}^{\prime}=-4.8$ are available in our sample. A similar trend appears in the dependence of $\alpha / \sqrt{\sin i}$ on $\log L_{\mathrm{X}} / L_{\mathrm{bol}}$ in Fig. 18b; while over the whole interval of X-ray luminosities rigid rotators are present, for a subgroup of stars in the region $-5.6<\log L_{\mathrm{X}} / L_{\mathrm{bol}}<-4.6, \alpha / \sqrt{\sin i}$ may depend on the normalized X-ray flux.

Our data indicates that especially the chromospherically most active stars tend to be rigid rotators. Henry et al. (1996) place a transition region between active and incative stars at $\log R_{\mathrm{HK}}^{\prime}=-4.75$. These authors believe that stars spend a third of their lifetime in the active stage and remain inactive after crossing the transition region. Our results are severely limited by the extent of coinciding measurements of differential rotation and activity indicators, and in particular nothing can be said about the rotation law of stars with $\log R_{\mathrm{HK}}^{\prime}<-4.8$. Although the results from our small sample remain preliminary, stars in the active phase seem to be rigid rotators while those in the transition region do show evidence for differential rotation. On the basis of our data we cannot yet decide whether differential rotation is a property only of the inactive stars belonging to the transition region, or whether it is just a reflection of the properties of our sample.

What makes this even more interesting is the dependence of the ratio $\log \left(\omega_{\mathrm{cyc}} / \Omega\right)$ on $\log R_{\mathrm{HK}}^{\prime}$, with $\omega_{\mathrm{cyc}}$ denoting the dynamo cycle frequency and $\Omega$ the rotational frequency. Brandenburg et al. (1998) found two distinct branches of active and inactive stars in their Fig. 2 with a jump between them at values of $\log R_{\mathrm{HK}}^{\prime} \simeq-4.75$. Our measurements favour the onset of differential rotation in the transition region, but, again, no statement is possible about the dominating group of inactive stars from the $\log R_{\mathrm{HK}}^{\prime}$ values of our sample.

A possible way to overcome this problem is to search for similar regions in the X-ray data. Sterzik \& Schmitt (1997) found a linear correlation between the chromospherically emission in terms of $\log R_{\mathrm{HK}}^{\prime}$ and X-ray to bolometric luminosity $\log L_{\mathrm{X}} / L_{\mathrm{bol}}$. Naturally the scatter in such relations is quite large since values of $\log R_{\mathrm{HK}}^{\prime}$ are mean values measured over years while $\log L_{\mathrm{X}} / L_{\mathrm{bol}}$ mostly comes from a snapshot. Nevertheless, according to the relation found by Sterzik \& Schmitt (1997) the transition region at $\log R_{\mathrm{HK}}^{\prime} \simeq-4.75$ should be found at $\log L_{\mathrm{X}} / L_{\mathrm{bol}} \simeq-5.6$. However, no indication for a similar transition in the values of $L_{\mathrm{X}} / L_{\mathrm{bol}}$ was actually apparent in their data.

In Fig. 18b it appears that the region where differential rotation in our sample sets in, does not show up at a value of $\log L_{\mathrm{X}} / L_{\mathrm{bol}} \simeq-5.6$, but at around $\log L_{\mathrm{X}} / L_{\mathrm{bol}} \simeq-5.2$. At the top panel of Fig. $18 \mathrm{~b}$ we have indicated the values of $\log R_{\mathrm{HK}}^{\prime}\left(\log L_{\mathrm{X}} / L_{\mathrm{bol}}\right)$, i.e., the values of chromospheric activity derived from the X-ray to bolometric luminosity following the relation given in Sterzik \& Schmitt (1997). Reading Fig. 18b as a $\alpha / \sqrt{\sin i}$ vs. $\log R_{\mathrm{HK}}^{\prime}$ plot a comparison to Fig. 18a reveals a similar overall picture but with an offset in the direction of $\log R_{\mathrm{HK}}^{\prime}$. It remains unclear whether the $\log R_{\mathrm{HK}}^{\prime}-\log L_{\mathrm{X}} / L_{\mathrm{bol}}$ relation is systematically shifted for our subgroup of stars (e.g., differential rotators or stars in the transition region) or whether the offset simply is an effect due to our limited sample and the intrinsic scatter of the X-ray snapshots.

To conclude, the correlations of $\alpha / \sqrt{\sin i}$ to both activity indicators, $\log R_{\mathrm{HK}}^{\prime}$ and $\log L_{\mathrm{X}} / L_{\mathrm{bol}}$, in our sample reveal a clustering of differential rotators in a relatively well defined stage of activity. Whether this clustering reflects the onset of differential rotation during a star's transfer from an active to an inactive stage cannot be answered from our sample.

\section{7. $\Delta \Omega$ and $\Delta P$}

In this subsection we will investigate the dependence of the often used parameters $\Delta \Omega$ and $\Delta P$ on $\Omega$ and $P$, respectively. Given the radius $R$ from their positions in the HR-D (Cox 2000), $\Delta \Omega$ and $\Delta P$ can easily be calculated from $\alpha=\Delta \Omega / \Omega$ and $P=2 \pi / \Omega$. We focus on the stars with significant evidence 


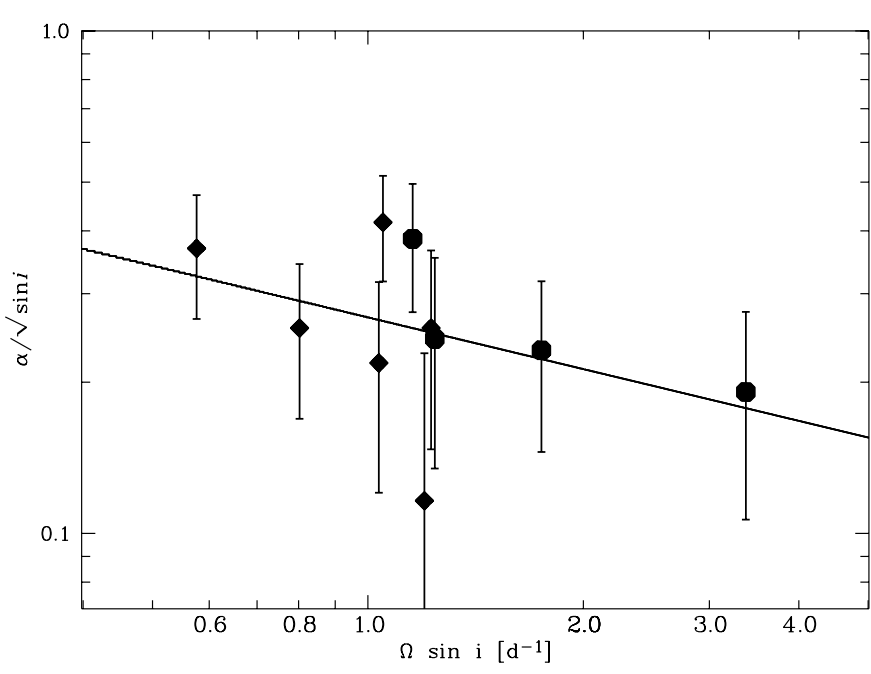

Fig. 19. Differential rotation $\alpha / \sqrt{\sin i}$ vs. angular velocity $\Omega \sin i$. Stars of later type $(B-V>0.46)$ are plotted with squares, early types with values of $B-V \leq 0.46$ with circles. The result of a formal regression analysis is plotted over the data.

for differential rotation, i.e., we assume that a threshold divides the differential from the rigid rotation regime. Although we do not know what triggers the onset of differential rotation, we search for dependences only among the stars with significant evidence for differential rotation.

It is important to note that the uncertainties in the values of $\alpha$ inherent in FTM do not allow us to detect differential rotation of the amount $\alpha \lesssim 10 \%$ independent of the value of $v \sin i$. Thus we are not able to detect differential rotation rates as low as claimed for example from Doppler images for the K0 dwarf AB Dor $\left(\alpha=\Delta \Omega / \Omega \simeq 4.6 \times 10^{-3}\right.$, Donati \& Collier Cameron 1997). Simulations of the angular velocity dependence of differential rotation were carried out for a G2 and a K5 dwarf by Kitchatinov \& Rüdiger (1999). Motivated by results from observational works they searched for a power law dependence of the form

$\frac{\Delta \Omega}{\Omega} \propto \Omega^{-n^{\prime}}$.

Kitchatinov \& Rüdiger (1999) found that $n^{\prime}$ is not a constant but varies in the region $0.95 \lesssim n^{\prime} \lesssim 1.56$ with a mean value of $n^{\prime}=1.15$ for G2. Furthermore they show that the amount of $\alpha$ is significantly larger for the spectral type G2 than for K5 while the dependence on angular velocity is comparable for both spectral types. No simulations for F-stars were carried out but it seems not implausible to assume that for F-stars a similar angular velocity dependence remains valid.

Our values of $\alpha=\Delta \Omega / \Omega$ that are significantly larger than 0.0 , i.e., $\alpha-\delta \alpha>0.0$, are plotted against $\Omega \sin i$ in Fig. 19 . A rough decrease in $\alpha$ with increasing $\Omega \sin i$ is apparent, although the scatter is quite large. A formal regression analysis to Eq. (7) yields

$$
\frac{1}{\sqrt{\sin i}} \cdot \frac{\Delta \Omega}{\Omega}=(0.27 \pm 0.03)(\Omega \sin i)^{-0.34 \pm 0.26} \text {. }
$$

Obviously, although the errors of the regression curve are large, our derived $n^{\prime}=0.34 \pm 0.26$ is inconsistent with the values predicted by Kitchatinov \& Rüdiger (1999). As already pointed

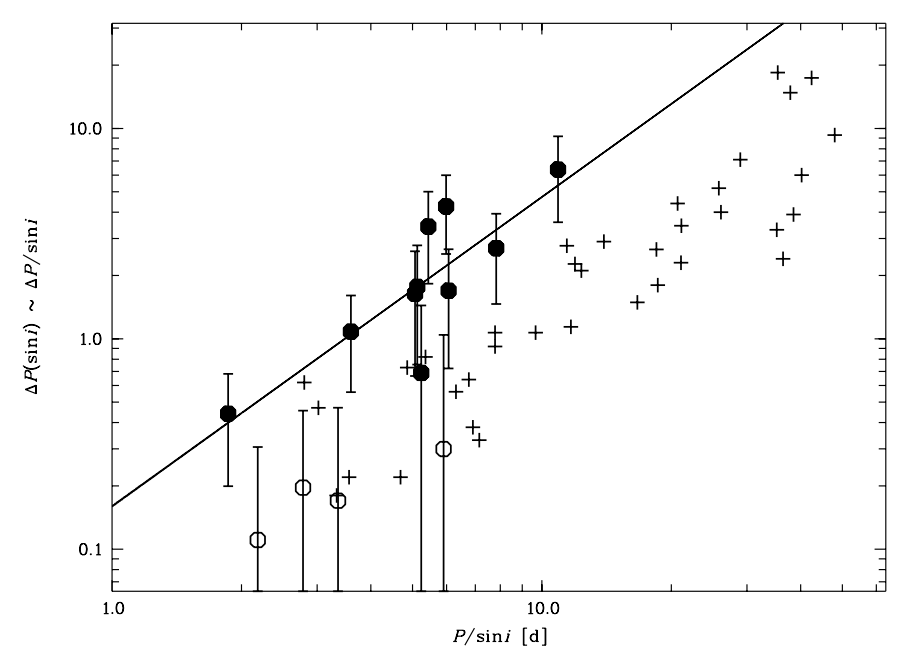

Fig. 20. Values of $\Delta P(\sin i) \simeq \Delta P / \sin i$ derived from Eq. (10) vs. $P / \sin i$ for the stars of our sample that show differential rotation. Open circles represent stars that are consistent with rigid rotation. The solid line is a fit to the values from stars not consistent with rigid rotation (full circles). Crosses represent data from Donahue et al. (1996).

out in that work, such disagreement may be partly due to our sample's combined different spectral types. The highest angular velocities are mainly found for early F-stars (circles in Fig. 19), the lowest ones on late F-stars (squares in Fig. 19). Since models suggest that later spectral types have lower differential rotation rates, combining different spectral types would indeed reduce the slope $n^{\prime}$.

The observational results Kitchatinov \& Rüdiger (1999) compared their model to were derived from the chromosperic Ca II H and K lines. Donahue et al. (1996) search for differential rotation by measuring variations in seasonal rotational periods; assuming that active regions migrate over the stellar surface in analogy to the solar case, acceleration towards the equator would diminish the length of the rotational period during a stellar cycle. Donahue et al. (1996) searched for a period dependent range $\Delta P$ in the observed period of the form

$\Delta P \propto P^{n^{\prime \prime}}$

and found $\Delta P \propto<P>^{1.3 \pm 0.1}$. The authors point out that in analogy to the solar case active regions may not reach over all latitudes and the observed period change may reflect only a part of the period variation; thus absolute values of $\Delta P$ may in fact be higher. However, assuming comparable latitudes of activity for different spectral types, the value of $n^{\prime \prime}=1.3 \pm 0.1$ should be valid independent of the absolute values of $\Delta P$.

For a direct comparison we derived values of $\Delta P$ for the stars of our sample using the equation

$\Delta P=P \frac{\alpha}{1-\alpha}$

Since we measured only $v \sin i$ instead of the pure rotational velocity $v$, we only have the measured values $\alpha / \sqrt{\sin i}$ and $P / \sin i$ instead of $\alpha$ and $P$. The inclination dependence of $\Delta P(\sin i)$ is 
a bit more complicated. Evaluating Eq. (10) with the measured quantities including $v \sin i$, we obtain

$$
\begin{aligned}
\Delta P(\sin i) & =\frac{P}{\sin i} \frac{\alpha}{\sqrt{\sin i}\left(1-\frac{\alpha}{\sqrt{\sin i}}\right)} \\
& =\frac{P}{\sin i} \frac{\alpha}{\sqrt{\sin i}-\alpha} .
\end{aligned}
$$

For sufficiently large inclination angles we approximate

$$
\frac{\alpha}{\sqrt{\sin i}-\alpha} \simeq \frac{\alpha}{1-\alpha}
$$

and are thus led to plot our measurements of $\Delta P(\sin i)$ vs. $P / \sin i$ in Fig. 20 (full circles). For comparison we overplotted the values of $\Delta P$ from $\mathrm{Ca}$ II $\mathrm{H}$ and $\mathrm{K}$ lines given in Donahue et al. (1996) (crosses). The open circles represent four of our measurements that are consistent with solid rotation but have a value of $\alpha>0.0$. Regression analysis for the significant measurements of differential rotation (excluding the four values consistent with rigid rotation) yields

$\Delta \frac{P}{\sin i}=10^{(-0.80 \pm 0.26)}\left(\frac{P}{\sin i}\right)^{(1.47 \pm 0.35)}$.

Thus we found in our sample a value of $n^{\prime \prime}=1.47 \pm 0.35$ in very good agreement with $n^{\prime \prime}=1.3 \pm 0.1$ derived by Donahue et al. (1996). This agreement describes what is apparent in Fig. 20; although there is an offset between our data and that from Ca II $\mathrm{H}$ and $\mathrm{K}$ lines, the slopes of both datasets are comparable.

As mentioned above, the values of $\Delta P$ measured by period variations in $\mathrm{Ca}$ II $\mathrm{H}$ and $\mathrm{K}$ lines must be interpreted as lower limits since the differential character can be obtained only along the active latitudes. Let us assume that the offset between our values of $\Delta P$ and the values from Donahue et al. (1996) is due to the limited sensitivity in latitude of the photometric method. Let us further assume that active regions appear and migrate in analogy to the solar case and that the stellar rotational law is solar-like and of the form $\Omega(l)=\Omega_{0}\left(1-\alpha \sin ^{2} l\right)$ with $l$ being the latitude. Then we can estimate the highest latitudes where stellar active regions appear in the early phase of a stellar magnetic cycle (or where they induce photometric signatures in $\mathrm{Ca}$ II $\mathrm{H}$ and $\mathrm{K}$ ). This highest latitude $l_{\max }$ can be calculated from

$\sin ^{2} l_{\max }=\frac{\Omega}{X(\Omega-\Delta \Omega)+\Delta \Omega}$

with the measured ratio of

$X=\frac{\Delta P_{\text {this work }}}{\Delta P_{\text {Donahue et al. }}}=2.7_{-1.5}^{+2.3}$

and Eq. (8). For $\Omega>0.5 \mathrm{~d}^{-1}$, Eq. (13) depends only slightly on the value of $\Omega$. Neglecting possible inclination angles of $i<90^{\circ}$ in Eq. (8), we derive for all $\Omega>0.5 \mathrm{~d}^{-1}$ a maximum latitude where active regions emerge on the stellar surfaces of

$l_{\max }=\left(40_{-10}^{+30}\right)^{\circ}$,

a value in surprisingly good agreement with the active regions from the Sun.

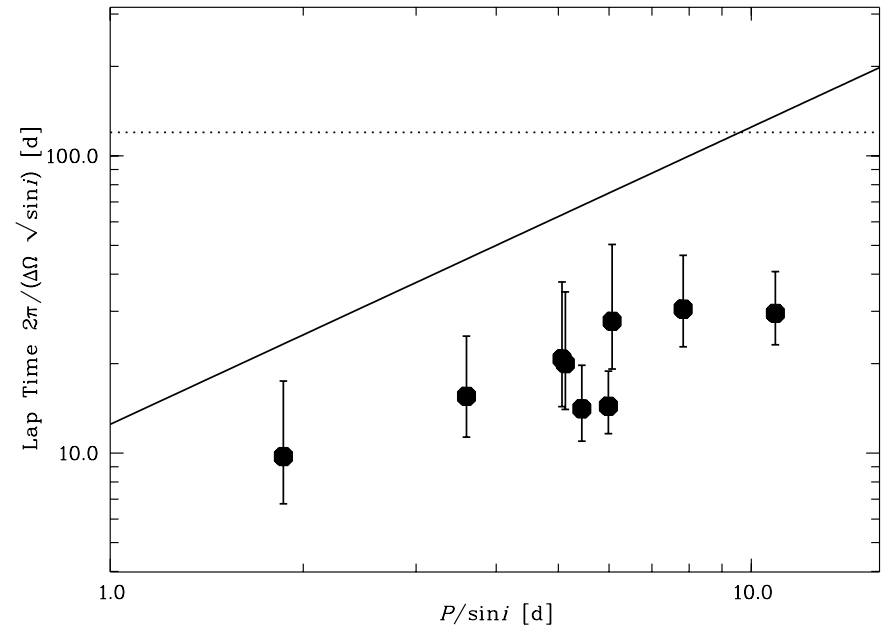

Fig. 21. Lap time $2 \pi /(\Delta \Omega \sqrt{\sin i})$ plotted against $P / \sin i$. The solar value of $2 \pi / \Delta \Omega \simeq 130 \mathrm{~d}$ is indicated with a dashed line. Due to the limited sensitivity of FTM, lap times of that order can only be detected for the slowest rotators. The period dependent maximum value of the detectable lap time is shown with a solid line.

We want to emphasize that the interpretation of the offset between data from chromospheric $\mathrm{Ca}$ II $\mathrm{H}$ and $\mathrm{K}$ period variations and from our FTM analysis is somewhat speculative and that many uncertainties arise during the different derivations. Even some simplifications in our regression analysis, e.g., neglecting the stars with $\alpha=0.0$, lack physical motivations and need further investigation by the examination of more objects. Nevertheless, the coincidence of the derived maximum active latitudes $l_{\max }$ with the solar case is an encouraging result that points towards a possible connection between differential rotation measurements from photometry and FTM.

To be complete we calculated the lap times $2 \pi / \Delta \Omega$, i.e., the time the equatorial regions need to lap the pole. We plot them vs. the rotational period $P / \sin i$ in Fig. $21.2 \pi / \Delta \Omega$ is often used as an indicator for dynamo efficieny. Results from Doppler imaging suggest that $2 \pi / \Delta \Omega$ is a constant for stars of different rotational periods and close to the solar value of $130 \mathrm{~d}$, e.g., 110 d for AB Dor (Donati \& Collier Cameron 1997).

The dotted line in Fig. 21 indicates the solar value of $2 \pi / \Delta \Omega \simeq 130 \mathrm{~d}$. Our measured values from the stars with detected differential rotation are significantly lower than that value. We note again that with FTM detections of differential rotation imply $\alpha \gtrsim 10 \%$; since $2 \pi / \Delta \Omega=P / \alpha$, we are limited to a period dependent value of the maximum lap time that can be detected with FTM. That maximum lap time is indicated as a solid line in Fig. 21. Although we can thus neither confirm nor disprove the occurence of differential rotators with lap times similar to the solar value we actually found some stars with evidence for significantly lower values of $2 \pi / \Delta \Omega$.

\section{Summary}

We measured the projected rotational velocities $v \sin i$ for a sample of 142 F-, G- and K-stars. For 32 stars of our sample the amount of differential rotation $\alpha=\left(\Omega_{\text {Equator }}-\Omega_{\text {Pole }}\right) / \Omega_{\text {Equator }}$ has been derived. We demonstrated our technique with that 
overall broadening profiles with extremely high signal-to-noise ratios can be obtained and correlated our results with stellar parameters from the literature. The conclusions of our paper can be summarized as follows:

1. The distributions of the rotational velocities and the line profile asymmetries of our sample stars are in agreement with expectations from the rotation and granulation boundary in the HR-diagram.

2. Evidence for differential rotation has been obtained for stars between 12 and $45 \mathrm{~km} \mathrm{~s}^{-1}$. Although differential rotation is more common in slowly rotating stars, no upper limit in $v \sin i$ is found for the occurence of differential rotation.

3. We found no correlations between differential rotation $\alpha$ and (i) mass or (ii) age. Fast rotating Li-depleted stars with evidence for differential rotation show no indication of high age. The interpretation that $\mathrm{Li}$-depletion in fast rotators is connected with internal mixing is supported.

4. Some indications exist that differential rotation is not a common phenomenon in very active stars with $\log R_{\mathrm{HK}}^{\prime}>$ -4.65 . While we have no data for inactive stars with $R_{\mathrm{HK}}^{\prime}<$ -4.85 , stars populating the presumed transition region between an active and an inactive phase at $\log R_{\mathrm{HK}}^{\prime} \approx-4.75$ show strong evidence for differential rotation.

5. Our data suggest a period dependence of differential rotation of the form $\Delta P / P=P^{(0.47 \pm 0.35)}$. Assuming that differences to results from CaII emission measurements are due to the limited sensitivity latitude of that data, we derive a maximum latitude for active regions in good agreement with the solar value.

6. Although using FTM we could not measure lap times $2 \pi / \Delta \Omega$ of the order of the solar value of $\sim 130 \mathrm{~d}$, we found evidence for lap times significantly lower than that value. This finding contradicts the assumption that comparable lap times - maintaining comparable dynamo efficiencies should be found on different stars.

Acknowledgements. A.R. acknowledges financial support from Deutsche Forschungsgemeinschaft DFG-SCHM 1032/10-1.

\section{References}

Barnes, J. R., Collier Cameron, A., James, D. J., \& Donati, J.-F. 2000, MNRAS, 314, 162

Balachandran, S. 1990, ApJ, 354, 310

Baliunas, S. L., Donahue, R. A., Soon, J. H., et al. 1995, ApJ, 438, 269

Bernacca, P. L., \& Perinotto, M. 1970-1973, Contrib. Oss. Asiago Nos., 239, 250, 294

Boesgaard, A. M., \& Tripicco, M. J. 1986, ApJ, 303, 724

Boesgaard, A. M., \& Lavery, R. J. 1986, ApJ, 309, 770

Brandenburg, A., Saar, S. H., \& Turpin, C. R. 1998, ApJ, 498, L51

Chen, Y. Q., Nissen, P. E., Benoni, T., \& Zhao, G. 2001, A\&A, 371, 943
Cox, A. N., ed. 2000, Allen's Astrophysical Quantities (AIP Press, Springer Verlag, New York)

Cutispoto, G., Pastori, L., de Medeiros, J. R., Tagliaferri, G., \& Andersen, J. 2002, A\&A, 384, 491

Donahue, R. A., Saar, S. H., \& Baliunas, S. L. 1996, ApJ, 466, 384

Donati, J.-F., Cameron, A. 1997, MNRAS, 291, 1

Dravins, D., Lindegren, L., \& Torkelsson, U. 1990, A\&A, 237, 137

ESA 1997, The Hipparcos and Tycho Catalogues, ESA SP-1200

Fekel, F. C. 1997, PASP, 109, 514

Groot, P. J., Piters, A. J. M., \& van Paradijs, J. 1996, A\&AS, 118, 545

Gray, D. F. 1973, ApJ, 184, 161

Gray, D. F. 1977, ApJ, 211, 198

Gray, D. F. 1982, ApJ, 258, 201

Gray, D. F. 1988, Lectures on spectral-line analysis: F, G and K stars (The Publisher, Arva)

Gray, D. F. 1991, Dynamo action in evolved stars, ed. I. Tuominen, D. Moss, \& G. Rüdiger, IAU Coll. 130, The Sun and Cool Stars: Activity, Magnetism, Dynamos (Springer, Berlin), 336

Hall, D. S. 1991, in The Sun and Cool stars, activity, magnetism, dynamos, ed. I. Tuominen, D. Moss, \& G. Rüdiger (Springer Verlag, New York), 353

Hauck, B., \& Mermilliod, M. 1998, A\&AS, 129, 431

Henry, T. J., Soderblom, D. R., Donahue, R. A., \& Baliunas, S. L. 1996, ApJ, 111, 439

Hoffleit, E. D., \& Warren Jr., W. H. 1991, The Bright Star Catalogue, 5th Revised Ed.

Hünsch, M., Schmitt, J. H. M. M., \& Voges, W. 1998, A\&AS, 132, 155

Hünsch, M., Schmitt, J. H. M. M., Sterzik, M. F., \& Voges, W. 1998, A\&AS, 135,319

Kitchatinov, L.L., \& Rüdiger, G. 1999, A\&A, 344, 911

Kupka, F., Piskunov, N. E., Ryabchikova, T. A., Stempels, H. C., \& Weiss, W. W. 1999, A\&AS, 138, 119

Lang, K. R. 1992, Astrophysical Data: Planets and Stars (SpringerVerlag, Berlin, Heidelberg, New York)

Moon, T. T. 1985, Coom. Univ. London Obs., 78

Moon, T. T., \& Dworetsky, M. M. 1985, MNRAS, 217, 305

Napiwotzki, R., Schönberger, D., \& Wenske, V. 1993, A\&A, 268, 653

Noyes, R. W., Hartmann, L. W., Baliunas, S. L., Duncan, D. K., \& Vaughan, A. H. 1984, ApJ, 279, 763

Pallavicini, R., Golub, L., Rosner, R., et al. 1981, ApJ, 248, 279

Reed, B. C. 1998, J. R. Astron. Soc. Can., 92, 36

Reiners, A., Schmitt, J. H. M. M., \& Kürster, M. 2001, A\&A 376, L13

Reiners, A., \& Schmitt, J. H. M. M. 2002a, A\&A, 384, 555

Reiners, A., \& Schmitt, J. H. M. M. 2002b, A\&A, 388, 1120

Reiners, A., \& Schmitt, J. H. M. M. 2002c, Poster Proceedings of the 1st Potsdam Thinkshop on Sunspots \& Starspots, Potsdam, ed. K. G. Strassmeier, \& A. Washuettl, 5

Reiners, A., \& Schmitt, J. H. M. M. 2002d, A\&A 393, L77

Saar, S. H., \& Osten, R. A. 1997, MNRAS, 284, 803

Siess, L., Dufour, E., \& Forestini, M. 2000, A\&A, 358, 593

Sterzik, M. F., \& Schmitt, J. H. M. M. 1997, AJ, 114, 167

Uesugi, A., \& Fukuda, I. 1982, Revised Catalogue of Rotational Velocities, Department of Astronomy, Kyito Univ., Japan

Wöhl, H. 1983, in Solar and Stellar Magnetic Fields: Origin and Coronal Effects, ed. J. O. Stenflo (Reidel Dordrecht), IAU Symp., 102,155 